\title{
A Priori Analysis of a Compressible Flamelet Model using RANS Data for a Dual-Mode Scramjet Combustor
}

\author{
Jesse R. Quinlan* and Tomasz G. Drozda ${ }^{\dagger}$ \\ NASA Langley Research Center, Hampton, VA, 23681 \\ James C. McDaniel ${ }^{\ddagger}$ \\ University of Virginia, Charlottesville, VA, 22904 \\ Guilhem Lacaze ${ }^{\S}$ and Joseph Oefelein \\ Sandia National Laboratories, Livermore, CA, 94550
}

\begin{abstract}
In an effort to make large eddy simulation of hydrocarbon-fueled scramjet combustors more computationally accessible using realistic chemical reaction mechanisms, a compressible flamelet/progress variable (FPV) model was proposed that extends current FPV model formulations to high-speed, compressible flows. Development of this model relied on observations garnered from an a priori analysis of the Reynolds-Averaged Navier-Stokes (RANS) data obtained for the Hypersonic International Flight Research and Experimentation (HIFiRE) dual-mode scramjet combustor. The RANS data were obtained using a reduced chemical mechanism for the combustion of a JP-7 surrogate and were validated using available experimental data. These RANS data were then post-processed to obtain, in an $a$ priori fashion, the scalar fields corresponding to an FPV-based modeling approach. In the current work, in addition to the proposed compressible flamelet model, a standard incompressible FPV model was also considered. Several candidate progress variables were investigated for their ability to recover static temperature and major and minor product species. The effects of pressure and temperature on the tabulated progress variable source term were characterized, and model coupling terms embedded in the Reynoldsaveraged Navier-Stokes equations were studied. Finally, results for the novel compressible flamelet/progress variable model were presented to demonstrate the improvement attained by modeling the effects of pressure and flamelet boundary conditions on the combustion.
\end{abstract}

\section{Introduction}

The extension of incompressible flamelet/progress variable (IFPV) models ${ }^{1,2}$ to high-speed, compressible, turbulent combustion has been the subject of numerous research efforts ${ }^{3-5}$ in recent years. Such research is fueled in part by the need to reduce the computational cost of performing reacting flow simulations of high-speed combustors of engineering complexity. While alternative approaches to reduce computational cost may be found in the literature, such as in-situ adaptive tabulation (ISAT), ${ }^{6}$ skeletal mechanisms, ${ }^{7}$ and eddy breakup models, ${ }^{8}$ to cite a few, several benefits of flamelet approaches warrant their use. Specifically, since all thermochemical data are computed in a preprocessing step and subsequently tabulated, the large number of species and reactions comprising detailed reaction mechanisms has little effect on the total simulation cost. In fact, only transport equations for the scalars used in the model parameterization, in addition to the Navier-Stokes equations, require computation. Additionally, with the appropriate parameterization of

\footnotetext{
*Aerospace Engineer, Pathways Program, Aeronautics Systems Analysis Branch, 1 N Dryden Street, and AIAA Member.

$\dagger$ Aerospace Engineer, Hypersonic Airbreathing and Propulsion Branch, 22 Langley Blvd, and AIAA Senior Member.

¥Professor, Department of Mechanical and Aerospace Engineering, 122 Engineer's Way, and AIAA Associate Fellow.

$\S$ Member of the Technical Staff, Combustion Research Facility, 7011 East Avenue, and AIAA Member.

๑Distinguished Member of the Technical Staff, Combustion Research Facility, 7011 East Avenue, and AIAA Associate Fellow.
} 
the thermochemical state-space, a flamelet/progress variable (FPV) model may recover finite-rate effects, extinction and reignition, compressibility, and non-adiabatic heat losses. Many of these parameterizations were previously developed in the context of incompressible combustion and further research is needed to adopt them to compressible flows. Moreover, compressible combustion introduces new modeling challenges that must be addressed.

The current study addresses several of the challenges facing a compressible flamelet/progress variable (CFPV) model by following a unique a priori approach, termed a priori flamelet-modeled RANS (APFMRANS), and applying it to the HIFiRE Direct Connect Rig (HDCR) combustor data. ${ }^{9}$ This study builds upon a previously-reported a priori analysis of the HDCR data. ${ }^{10,11}$ Within the proposed APFM-RANS analysis, a flamelet table is first constructed by solving the flamelet equations and is subsequently parameterized by a small set of tracking scalars, including mixture fraction, progress variable, and other scalars depending upon the flamelet model formulation. These tracking scalars of interest are computed from the RANS simulations of the HDCR that utilized finite-rate chemical kinetics and are then used to retrieve other necessary thermochemical data from the flamelet table. The retrieved data consists of the species mass fractions, which are used together with the energy and pressure to calculate the temperature; the species productions rates, which are used to construct the progress variable production rate; and the energy interdiffusion terms, which are used to close the energy equation.

In the current work, the above outlined analysis was used to perform a trade study on the efficacy of several candidate progress variables found in the literature. The use of pressure as a means of accounting for compressibility effects on the combustion was investigated. Several numerically-efficient methods of constructing model coupling terms present in the governing equations for the total energy were also considered. Finally, the observations of the variability in the oxidizer temperature were used to propose a novel CFPV model formulation.

\section{Methodology}

Early flamelet models relied on parameterizing thermochemical state-spaces using mixture fraction, $Z$, and scalar dissipation rate, $\chi \cdot^{1,2}$ Pierce and Moin ${ }^{12}$ later demonstrated the benefits of using a progress variable, $C$, in place of $\chi$, and this FPV model formulation was widely adopted in the literature ${ }^{13-16}$ for simulating low-speed reacting flows using flamelet-based approaches. In this section, several FPV models and the transport equations solved by the Viscous Upwind aLgorithm for Complex flow ANalysis Comptuational Fluid Dynamics (VULCAN-CFD) ${ }^{17}$ code are discussed. In subsequent sections, the APFM-RANS analysis method is introduced, and the FPV model formulations used in this study are discussed. Finally, procedures for flamelet table generation and data retrieval methods are described.

\section{II.A. FPV Model Implementation Considerations}

The APFM-RANS analysis method was developed to efficiently study the modeling characteristics of a variety of compressible flamelet models. For reacting RANS calculations, the compressible Reynolds-averaged transport equations for mass, momentum, total energy, and reactive scalars are solved. This system of transport equations is closed using the ideal gas equation of state. For flamelet-based modeling approaches, however, all of the reactive scalar transport equations are replaced by several equations describing the transport of the tracking scalars used to parameterize the thermochemical state-space. This alternative system generally includes a single passive (typically mixture fraction, $Z$ ) and a single reactive scalar (typically a progress variable, $C$ ) transport equation of the form shown respectively in Eqs. (1) and (2), where $\rho$ is the mass density, $u_{j}$ are the convective velocities, $D$ is the mass diffusivity, $\dot{\omega}_{C}$ is the net production rate of $C$, $t$ is time, and $x_{j}$ are the space dimensions.

$$
\begin{gathered}
\frac{\partial \rho Z}{\partial t}+\frac{\partial \rho u_{j} Z}{\partial x_{j}}=\frac{\partial}{\partial x_{j}}\left(\rho D \frac{\partial Z}{\partial x_{j}}\right) \\
\frac{\partial \rho C}{\partial t}+\frac{\partial \rho u_{j} C}{\partial x_{j}}=\frac{\partial}{\partial x_{j}}\left(\rho D \frac{\partial C}{\partial x_{j}}\right)+\dot{\omega}_{C}
\end{gathered}
$$

To facilitate the ease of discussion, these transport equations are shown in their exact form. Time averaging (as in RANS) or spatially filtering (as in large-eddy simulation) these equations will introduce additional terms which will require closures. For simplicity of discussion, the closures for the scalar fluxes are assumed to 
take on a conventional gradient diffusion form. If turbulence-chemistry-interaction effects are to be modeled, additional transport equations may be necessary. However, these effects are not considered here so that any error associated with turbulence-chemistry-interaction modeling is not mistaken as FPV modeling error. In order to close Eq. (2), $\dot{\omega}_{C}$ must be calculated using the FPV model.

In order to close the transport equation for total energy, E, shown in Eq. (3) and the equation of state, shown in Eq. (4), the FPV model must be used to compute the species mass fractions, $Y_{\alpha}$. In these equations, $\tau_{i j}$ is the fluid stress tensor, $\lambda$ is the molecular thermal conductivity, $T$ is the static temperature, $h_{\alpha}$ are the species static enthalpies, $p$ is the static pressure, $R_{u}$ is the universal gas constant, $M W_{\alpha}$ are the species molecular weights, and $N$ is the total number of chemical species. In the context of FPV models, terms involving the species mass fractions must be computed by retrieving the latter from the flamelet table since those quantities are not transported.

$$
\begin{gathered}
\frac{\partial \rho E}{\partial t}+\frac{\partial \rho u_{j} E}{\partial x_{j}}=-\frac{\partial p u_{i}}{\partial x_{i}}+\frac{\partial \tau_{i j} u_{i}}{\partial x_{j}}+\frac{\partial}{\partial x_{j}}\left(\lambda \frac{\partial T}{\partial x_{j}}\right)+\underbrace{\frac{\partial}{\partial x_{j}}\left(\rho D \sum_{\alpha=1}^{N} h_{\alpha} \frac{\partial Y_{\alpha}}{\partial x_{j}}\right)}_{\beta} \\
p=\rho T R_{u} \sum_{\alpha=1}^{N} \frac{Y_{\alpha}}{M W_{\alpha}}
\end{gathered}
$$

For incompressible flow simulations with single air and fuel streams, the FPV models have been shown to work well to describe the thermochemical state for a range of problems of engineering interest. ${ }^{18-22}$ However, for a compressible flow, the static pressure cannot be assumed constant and may vary by an order of magnitude or more. The effect of $p$ on the chemical kinetics and composition must be considered within the FPV model. The proper way to account for this dependence requires adding $p$ to the flamelet library parameterization. When considering the implementation details of such a compressible FPV model, it is helpful to consider terms used to link the model to the governing equations, which include $Y_{\alpha}$, $\dot{\omega}_{C}$, and the energy interdiffusion term, $\beta$, marked above in Eq. (3). These linking terms will be considered in more detail in subsequent sections.

\section{II.B. APFM-RANS Anslysis Method and CFPVX Model Formulations}

The APFM-RANS analysis method was developed as a means to rapidly test candidate FPV model formulations in an a priori fashion for combustors of engineering complexity, such as the HDCR. Using this method along with data gathered during a previous a priori study, ${ }^{10}$ an FPV model accounting for both compressibility and flamelet boundary condition variation within the HDCR flowfield was developed. In this section, the APFM-RANS analysis method is outlined, and this new model, referred to as the CFPVX model, is described.

Application of the APFM-RANS analysis method relies on first performing a baseline RANS simulation of the flow using a finite-rate chemical kinetics reaction mechanism. This RANS solution serves as the truth dataset from which $Z$ and $C$ are computed and to which subsequent APFM-RANS analysis data are compared. Bilger's definition is used to calculate $Z$ for the current study. ${ }^{23}$ In a separate but concurrent step, a flamelet table is constructed by solving the flamelet equations. ${ }^{1}$ Details of the flamelet table generation are included in the following section. Once the $Z$ and $C$ fields are constructed from the RANS data and the flamelet table is complete, the desired FPV model formulation is used to compute an APFM-RANS solution.

Computing the APFM-RANS solution requires querying the flamelet table using the reconstructed FPV state vector, $\phi$, which for the IFPV model used for the current study consists of $\phi=[Z, C]^{T}$. Compressible models require additional parameters from the RANS solution, as described later. Linearly interpolated values for $Y_{\alpha}$ and $\dot{\omega}_{\alpha}$ are retrieved from the table based on $\phi$ and used to update the original RANS values. Using the updated $Y_{\alpha}$ and the internal energy from the RANS solution, $e_{R A N S}$, the APFM-RANS temperature, $T$, is calculated by performing a Newton-Raphson solve on Eq. (5), where $e_{R A N S}$ is the internal energy for the baseline RANS solution and $R$ is the mixture gas constant.

$$
0=e_{R A N S}-\sum_{\alpha=1}^{N} Y_{\alpha} h_{\alpha}(T)+T \sum_{\alpha=1}^{N} R \frac{Y_{\alpha}}{M W_{\alpha}}
$$


NASA thermodynamic polynomials were used to compute mixture static enthalpy, $h$, as a function of temperature during application of the APFM-RANS analysis method. ${ }^{24}$ Pressure can then be updated using the equation of state in Eq. (4). A flowchart detailing the APFM-RANS analysis method is included in Fig. 1.

In the current study, three FPV model formulations were tested. The first was the standard IFPV model developed by Pierce and Moin, ${ }^{12}$ in which the flamelet table was built by solving the flamelet equations over a range of $\chi_{s t}$ and subsequently mapping solutions to $Z$ and $C$ space. For this model, a single pressure level was used to compute all the flamelet solutions. Contrary to the standard IFPV model, $T$ was computed using the Netwon-Raphson solve on Eq. (5), rather than retrieving from the flamelet table. The second model was a CFPV model, in which the flamelet table was parameterized by $Z, C$, and $p$. Similar models can be found in the literature for application to diesel engines. ${ }^{3}$ For this model, a flamelet table was constructed by solving flamelet solutions across $\chi_{s t}$ and $p$, where baseline RANS data ${ }^{10}$ was used to identify physicallyrepresentative pressure levels. The third model tested using the APFM-RANS analysis method was a novel CFPV model in which flamelet oxidizer temperature was used as an additional parameterizing scalar. Using baseline RANS data as guidance, ${ }^{10}$ the flamelet table was built by solving the flamelet equations over physically-representative $\chi_{s t}, p$, and $T_{o x}$ and subsequently mapping the solutions to $Z, C, p$, and $h$. In this approach, $h$ was used as a proxy variable for $T_{o x}$. This model, referred hereafter as the CFPVX model, was designed to model the effects of both compressibility and varying flamelet boundary conditions on the combustion. This model is the first for which variation associated with flamelet boundary conditions, which in the current case is oxidizer temperature, is included in a Eulerian FPV model framework.

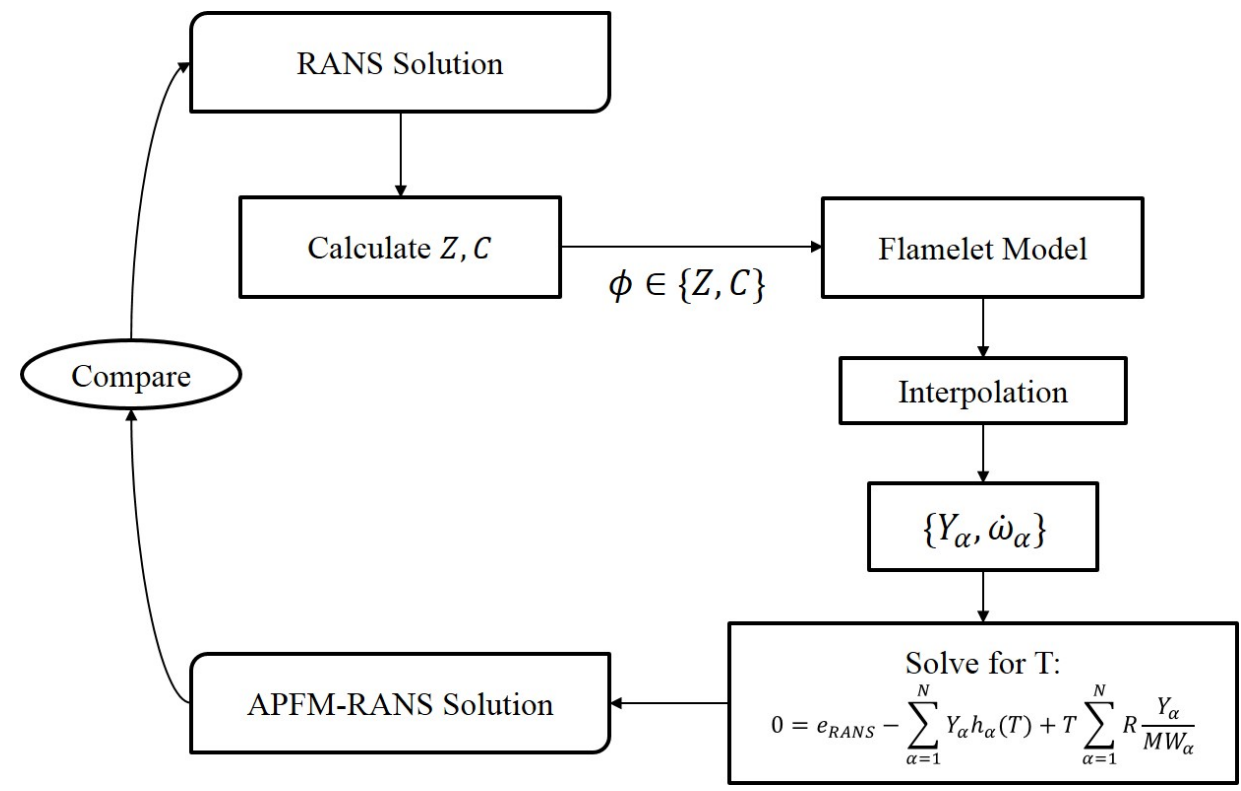

Figure 1. Flowchart depicting the APFM-RANS analysis method.

\section{II.C. Flamelet Table Generation}

A separate flamelet table was constructed for use with each of the three FPV models described in the previous section. For each table, the flamelet equations ${ }^{1}$ were solved using the FlameMaster ${ }^{25}$ software. These equations are shown below in Eqs. (6)-(7), where $\chi$ is the scalar dissipation rate, $\dot{\omega}_{\alpha}$ is the net production of species $\alpha$, and $c_{p}$ is the specific heat at constant pressure.

$$
\begin{gathered}
\frac{1}{2} \rho \chi \frac{\partial^{2} Y_{\alpha}}{\partial Z^{2}}+\dot{\omega}_{\alpha}=0 \\
\frac{1}{2} \rho \chi \frac{\partial^{2} T}{\partial Z^{2}}+\frac{1}{2} \frac{\rho \chi}{c_{p}} \frac{\partial c_{p}}{\partial Z} \frac{\partial T}{\partial Z}+\sum_{\alpha=1}^{N} \frac{1}{2} \frac{\rho \chi c_{p_{\alpha}}}{c_{p}} \frac{\partial Y_{\alpha}}{\partial Z} \frac{\partial T}{\partial Z}=\frac{1}{c_{p}} \sum_{\alpha=1}^{N} h_{\alpha} \dot{\omega}_{\alpha}
\end{gathered}
$$

The first table, for use with the IFPV model and hereafter referred to as FT1, consisted of 13 flamelet solutions computed at $p=1.0 \mathrm{~atm}$ and parameterized by $\chi_{s t}$, resulting in a two-dimensional state-space 
parameterized by $Z$ and $\chi_{s t}$. Fuel and oxidizer temperatures were held constant at $300 \mathrm{~K}$ and $1100 \mathrm{~K}$, respectively, which were set to approximately match the average fuel and oxidizer temperatures from the baseline RANS data.

The second table, hereafter referred to as FT2 and used for the CFPV model, consisted of 52 flamelet solutions at pressure levels including $p=0.5,2.5,4.5$, and $6.5 \mathrm{~atm}$. These $p$ levels were chosen to best represent the extent of pressure variation observed in the baseline RANS data of the HDCR for dual-mode operation. ${ }^{10}$ At each $p$ level, the solutions were parameterized by $\chi_{s t}$, resulting in a complete parameterization using $Z, \chi_{s t}$, and $p$, thereby yielding a three-dimensional state-space. Again, for FT2, the fuel and oxidizer temperatures were held constant at $300 \mathrm{~K}$ and $1100 \mathrm{~K}$, respectively.

The third flamelet table, hereafter referred to as FT3 and used for the CFPVX model, consisted of 256 flamelet solutions at pressure levels including $p=0.5,2.5,4.5$, and $6.5 \mathrm{~atm}$ and at oxidzer temperature levels including $T_{o x}=900,1100,1300,1500$, and $1700 \mathrm{~K}$. Similar to $p, T_{o x}$ levels were chosen to span the results of the baseline RANS data of the HDCR. ${ }^{10}$ These bounds were determined by examining the probability density function (PDF) for $T_{o x}$ for the baseline RANS data, which was computed by calculating $Z$ and sampling $T$ at points for $Z<0.01$. The PDF for $T_{o x}$ is shown in Fig. 2. Thus, the third table was parameterized by $Z, \chi_{s t}, p$, and $T_{o x}$, resulting in a four-dimensional state-space.

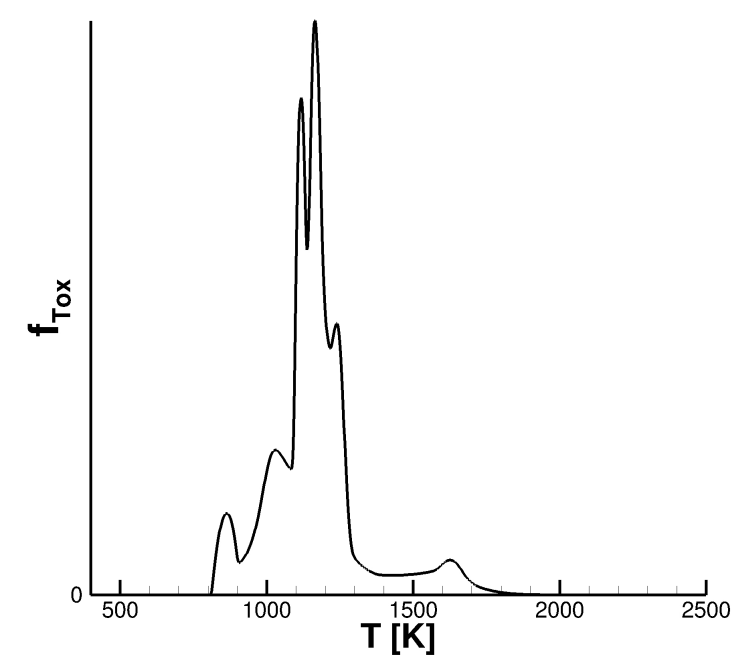

Figure 2. PDF of oxidizer temperature, $f_{T_{o x}}$, versus static temperature, $T$, for HDCR in dual-mode operation, including both the primary and secondary injector combustion regions.

Previous analysis of the HDCR combustor suggested the combustion data was characterized by a wide range of Damköhler number, which thereby supports the existence of strong finite-rate effects. ${ }^{10}$ For this reason, it was important to confirm that the FPV models and accompanying flamelet libraries included flamelet solutions along the unstable burning branch of the S-schaped curve, in addition to those along the mixing line and the branch of stable burning solutions. Without the inclusion of the unstable branch, transition from a burning state to the mixing line is non-physical, as demonstrated in Fig. 3. Therefore, each table included flamelet solutions completely traversing the underlying S-shaped curve, as shown by the S-curve for table FT1 in Fig. 4.

Since the FPV models used in this study employ a progress variable to model the reaction progress, the $\chi_{s t}$ was subsequently mapped to a corresponding $C$ for each of the three tables. Candidate $C$ definitions used for this study are discussed in the following section. In effect, FT1 comprised a two-dimensional state-space parameterized by $\phi=[Z, C]^{T}$, FT2 comprised a three-dimensional state-space parameterized by $\phi=[Z, C, p]^{T}$, and FT3 comprised a four-dimensional state-space parameterized by $\phi=\left[Z, C, p, T_{o x}\right]^{T}$. However, since $T_{o x}$ is not a transported variable in the VULCAN-CFD RANS framework, a further mapping was necessary. It was reasonable to posit that $h$ varied approximately monotonically with $T_{o x}$ for constant pressure and composition, or constant $Z, C$, and $p$, across the flamelet manifold due to the explicit dependence of $h$ on $T$ shown by the definition of $h$ in Eq. (8) for a mixture of thermally perfect gases, where $\Delta h_{f}^{o}$ is the mixture heat of formation, $T_{0}$ is the standard state temperature, and $c_{p_{\alpha}}$ is the specific heat at constant 


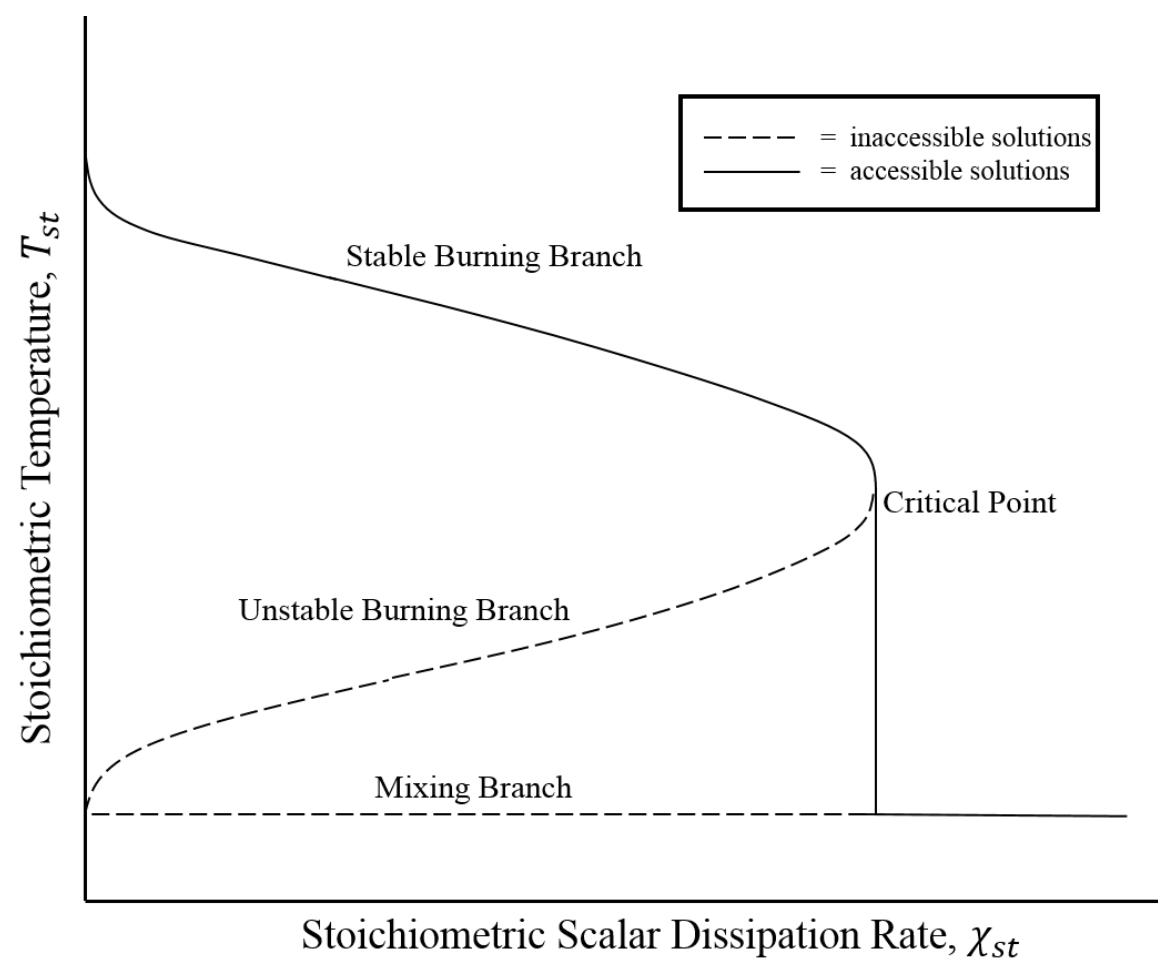

Figure 3. A notional S-shaped curve demonstrating the non-physical transition from the stable burning branch to the mixing line for flamelet libraries omitting the unstable solution branch. Dashed lines represent the region of flamelet solutions inaccessible to FPV models using $\chi_{s t}$ in place of $C$.

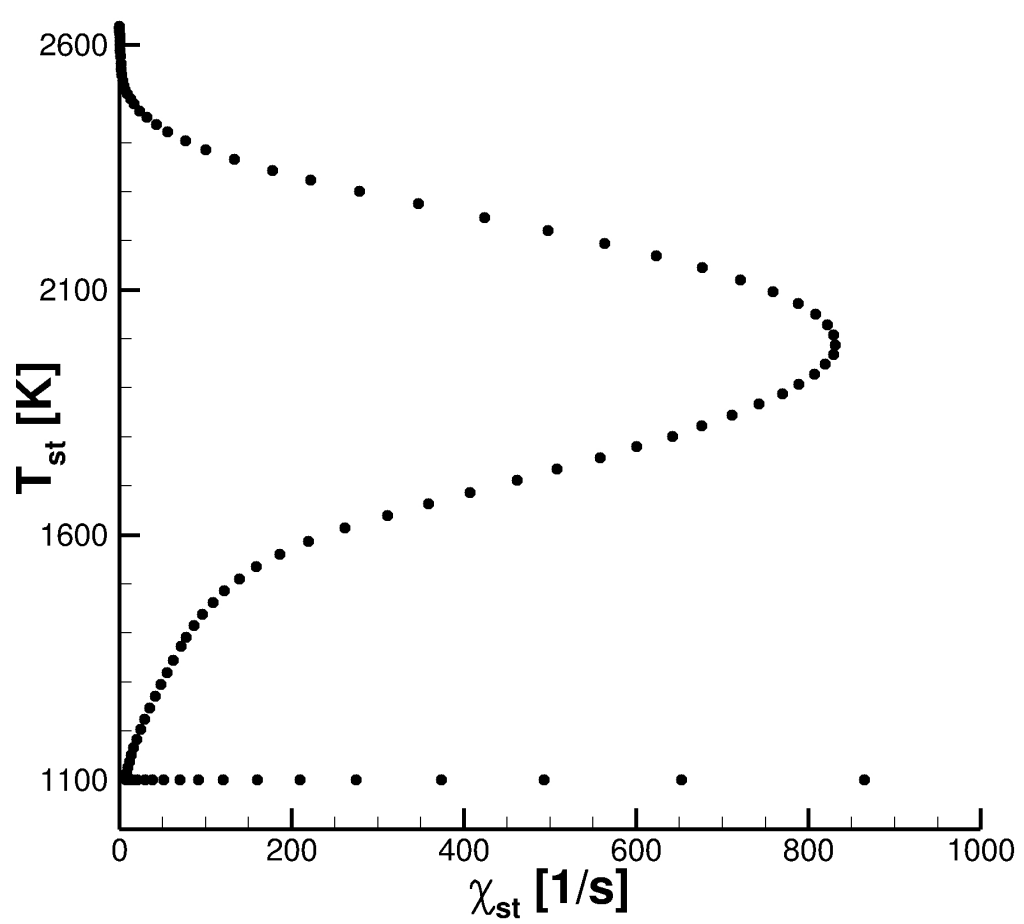

Figure 4. Stoichiometric temperature, $T_{s t}$, and scalar dissipation rate, $\chi_{s t}$, scatter plot for flamelet solutions $(\bullet)$ in table FT1 demonstrating the embedded S-shaped curve. 
pressure for species $\alpha$.

$$
h=\sum_{\alpha} h_{\alpha} Y_{\alpha}=\Delta h_{f}^{o}+\sum_{\alpha} Y_{\alpha} \int_{T_{0}}^{T} c_{p_{\alpha}} d T
$$

Thus, after building FT3 over $\phi=\left[Z, C, p, T_{o x}\right]^{T}$, the state-space was subsequently mapped to $\phi=[Z, C, p, h]^{T}$ for use with the CFPVX model. If turbulence-chemistry-interaction models were to be included, each table would be further parameterized by the mixture fraction and progress variable variances, thereby increasing the dimension of each table by two.

For each table, flamelet solution data for $Y_{\alpha}, \dot{\omega}_{\alpha}, T$, and $\frac{\partial Y_{\alpha}}{\partial Z}$ were stored for use by the APFM-RANS analysis method. The $\frac{\partial Y_{\alpha}}{\partial Z}$ terms are used to model the energy interdiffusion term in Eq. (3) and will be discussed in the next section. A set of FORTRAN routines were developed to build the flamelet tables from the FlameMaster solutions and to interface the flamelet table data with VULCAN-CFD and the APFMRANS software. Because the FlameMaster solutions are grid-adapted to the flame, and are therefore nonuniformly spaced, the analysis tools included binary search routines. In the case of higher-dimension flamelet tables, for which lookup times are considerably more expensive, an index-based search was also developed. In this approach, FlameMaster solutions are mapped onto a uniformly spaced flamelet state-space. The subsequent look up and interpolation are straight forward. The number of grid points in each dimension was then set to $100,40,10$, and 10 for $Z, C, p$, and $T_{o x}$, respectively, for the current study. For each call made to the table at runtime, the target $Z, C, p$, and $T_{o x}$ are converted to index representations on the uniform grid, and a quadrilinear interpolation is performed prior to returning the requested data. While this method results in a reduction in total lookup times, a uniform grid of sufficient resolution must be used in order to ensure interpolation errors remain on the same order as those of the grid-adapted solutions.

\section{Results}

The current study addresses several research objectives concerning the development and implementation of compressible FPV models. These objectives included: evaluating the efficacy of progress variable definitions available from the open literature for application to the HDCR combustor; gauging the effectiveness of pressure and temperature scaling techniques for recovering $\dot{\omega}_{C}$ from the flamelet table; evaluating model coupling terms embedded in the governing transport equations; and demonstrating the benefits of the proposed CFPVX model. The results for each of these objectives are presented in this section.

The baseline RANS case used for this study was performed for dual-mode operation of the HDCR, ${ }^{10}$ and the HDCR combustor moldline is included in Fig. 5. Both the primary and secondary injectors were fueled, for which $\Phi_{p}=0.15$ and $\Phi_{s}=0.5$, where $\Phi_{p}$ and $\Phi_{s}$ are the fuel equivalence ratios for the primary and secondary injectors, respectively. The plenum total temperature and total pressures were set to $1550 \mathrm{~K}$ and 14.63atm, respectively, to match a flight Mach number of 5.84. A quarter-geometry, structured grid was used for which the maximum y+ throughout the combustor never exceeded approximately 30.0, which allowed for the application of wall functions. ${ }^{26}$ Adiabatic surface boundary conditions prevented heat losses through the walls. The RANS equations were closed using the blended $k-\omega / k-\epsilon$ turbulence model of Menter. ${ }^{27}$ The low-dissipation flux split scheme of Edwards was used along with a monotone-upstream-centered scheme for conservation laws (MUSCL) with $\kappa$ of $1 / 3$, and the governing equations were integrated using an implicit diagonalized approximate factorization scheme with a CFL number of 2.0.

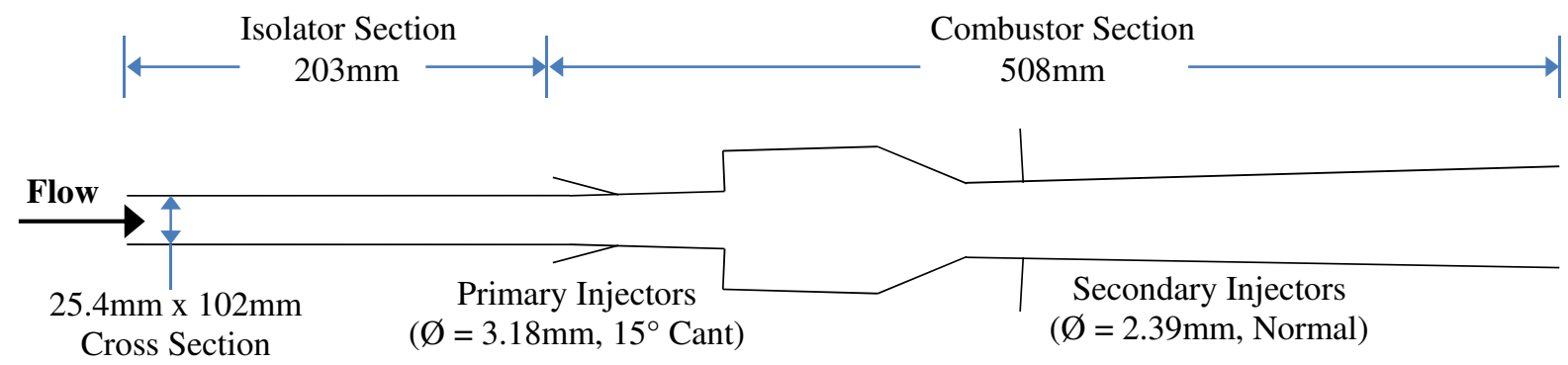

Figure 5. HDCR combustor moldline showing pertinent dimensions and locations of the primary and secondary injectors. 
A 22-species/18-step reduced chemical kinetics reaction mechanism ${ }^{28}$ originally designed for the combustion of ethylene was used to simulate the combustion of a mixture of $34 \% \mathrm{CH}_{4}$ and $64 \% \mathrm{C}_{2} \mathrm{H}_{4}$ by volume. Production rates were computed using the Reynolds-averaged $Y_{\alpha}, T$, and $p$, which is commonly referred to as the laminar chemistry assumption. As suggested by Storch et al. ${ }^{29}$, the turbulent Prandtl number and turbulent Schmidt number were set to 0.89 and 0.325 , respectively. The species mass diffusion coefficients were computed using constant laminar and turbulent Schmidt numbers.

\section{III.A. Progress Variable Trade Study}

In many applications of FPV models, $C$ is generally defined in an ad hoc fashion by the modeler in an attempt to best track the progress of the reaction system. In recent years, some attempts have been made by researchers to more rigorously define $C$ for a given fuel and reaction mechanism using optimization techniques. ${ }^{30,31}$ Motivated by the deficiencies highlighted by these efforts, a trade study was performed for several definitions of $C$ commonly used in the literature to model hydrocarbon fuel combustion, and the results are used to select the most appropriate functional form for simulations of the HDCR.

The most general requirement of $C$ is that it be defined such that it represents the global progress rate of the reaction system being modeled. Some formal requirements for $C$ were put forth by Ihme et al. ${ }^{30}$ The foremost of these is that all parameters defining the flamelet manifold uniquely identify each point in the thermochemical state-space. In this study, several commonly used formulations for $C$ from the literature were evaluated using the APFM-RANS analysis method for the dual-mode simulation of the HDCR. These progress variable definitions are shown in Eqs. (9), ${ }^{32-34}(10),{ }^{12}(11),{ }^{35,36}$ and $(12)^{37,38}$

$$
\begin{gathered}
C=Y_{\mathrm{CO}_{2}}+Y_{\mathrm{CO}} \\
C=Y_{\mathrm{CO}_{2}}+Y_{\mathrm{H}_{2} \mathrm{O}} \\
C=Y_{\mathrm{CO}_{2}}+Y_{\mathrm{H}_{2} \mathrm{O}}+Y_{\mathrm{CO}} \\
C=Y_{\mathrm{CO}_{2}}+Y_{\mathrm{H}_{2} \mathrm{O}}+Y_{\mathrm{CO}}+Y_{\mathrm{H}_{2}}
\end{gathered}
$$

where $Y_{\mathrm{CO}_{2}}, \mathrm{Y}_{\mathrm{CO}}, \mathrm{Y}_{\mathrm{H}_{2} \mathrm{O}}$, and $\mathrm{YOH}_{\mathrm{OH}}$ are mass fractions of $\mathrm{CO}_{2}, \mathrm{CO}, \mathrm{H}_{2} \mathrm{O}$, and $\mathrm{OH}$, respectively. For each of these progress variable definitions, APFM-RANS analyses were performed using IFPV, CFPV, and CFPVX models with flamelet tables FT1, FT2, and FT3, respectively. Twelve individual cases were analyzed for this study, and each will hereafter be referred to by its case ID. Each case ID identifies the progress variable and FPV model used. For example, case $I F P V 1$ refers to the APFM-RANS analysis for which the incompressible FPV model, $I F P V$, was used and where 1 indicates the first definition for $C$ in Eq. (9) was used. Similarly, case $C F P V X 4$ refers to the case in which the compressible FPVX model, $C F P V X$, was used and the fourth definition for $C$ in Eq. (12) was used. Additional details regarding these cases are included in Table 1. For the current study on $C$, results from cases IFPV1-CFPV4 were compared to those of the RANS solution using $T, Y_{\mathrm{CO}_{2}}, Y_{\mathrm{CO}}, Y_{\mathrm{H}_{2} \mathrm{O}}$, and $Y_{\mathrm{OH}}$. For each APFM-RANS analysis, the comparisons were made by first inspecting contours at the spanwise centerline of the first injector from the combustor centerline. Scatter plots of the APFM-RANS data were then constructed using the corresponding parameters from the RANS solution over the entire computational domain. 


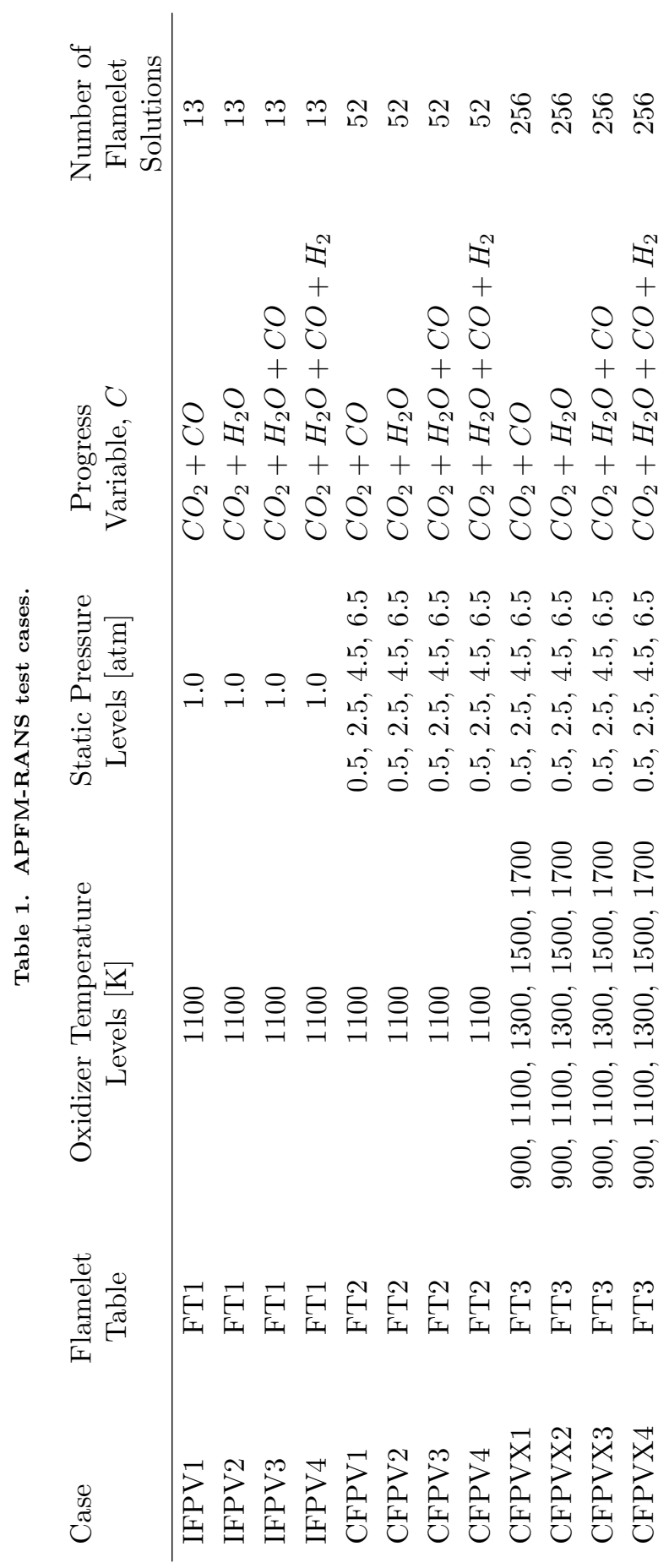

9 of 37

American Institute of Aeronautics and Astronautics 
Line plots of $T, Y_{\mathrm{CO}_{2}}, Y_{\mathrm{CO}}, Y_{\mathrm{H}_{2} \mathrm{O}}$, and $Y_{\mathrm{OH}}$ at selected streamwise locations along the injector centerline nearest to the combustor centerline are included for cases IFPV1-IFPV4 and cases CFPV1-CFPV4 in Figs. 610. For each of these figures, the RANS solution (truth model) is shown for comparison. For both the IFPV and CFPV models, the FPV-modeled solutions agree well with the baseline RANS solution for each of the comparison parameters. The line plots of $T, Y_{\mathrm{CO}_{2}}$, and $Y_{\mathrm{H}_{2}}$ obtained using the APFM-RANS analysis method agree well with those obtained from the baseline RANS simulations. The $Y_{C O}$ and $Y_{O H}$ fields exhibit weaker agreement with the baseline RANS solution for both the IFPV and CFPV models for all four $C$ definitions. While the trending of these parameters is similar to that of the RANS solution, it is clear that the FPV models under-predict the production of these minor species. Variation in agreement among the four candidate definitions for $C$ is less pronounced in these figures, and a more rigorous comparison must be subsequently made. However, it is clear already that the definition for $C$ given by Eq. (10) exhibits significant error due to the multivalued nature of the manifold in $Z$ - $C$-space around the stoichiometric curve, as seen in Fig. 8 at stations $25.5 \mathrm{~cm}$ and $42.0 \mathrm{~cm}$ for cases IFPV2 and CFPV2. The spikes in concentration of $Y_{C O}$ occur due to the multivaluedness of the flamelet manifold. 

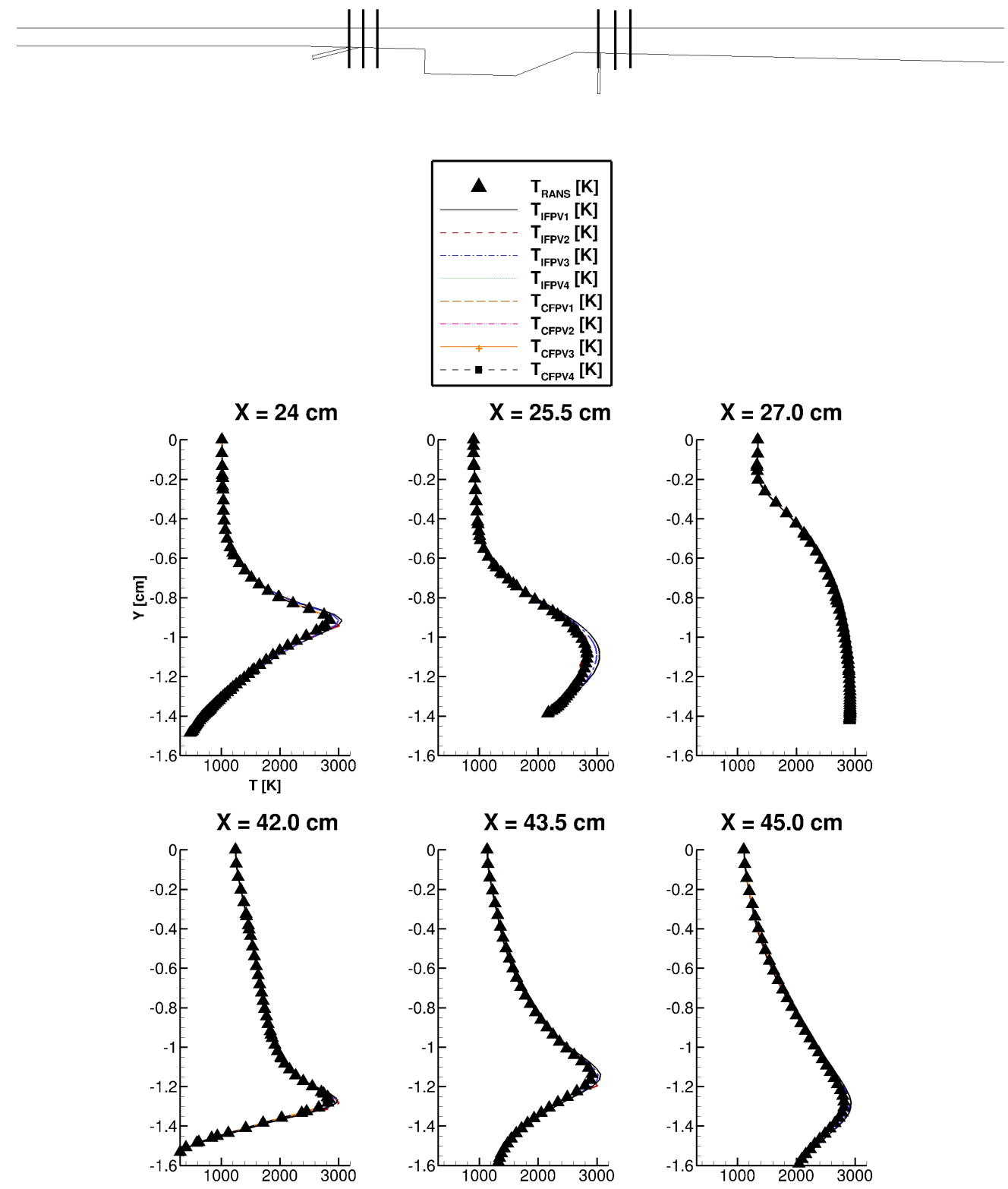

Figure 6. Line plots of $Y[\mathrm{~cm}]$ versus static temperature, $T[\mathrm{~K}]$, obtained using the APFM-RANS analysis method and from the baseline RANS simulations of the HDCR combustor at several locations through the primary and secondary injector flames at the injector centerline nearest to the combustor centerline for cases corresponding to IFPV1-IFPV4 and CFPV1-CFPV4. Units on each subfigure are the same as those shown on the top-row, left-most subfigure. 

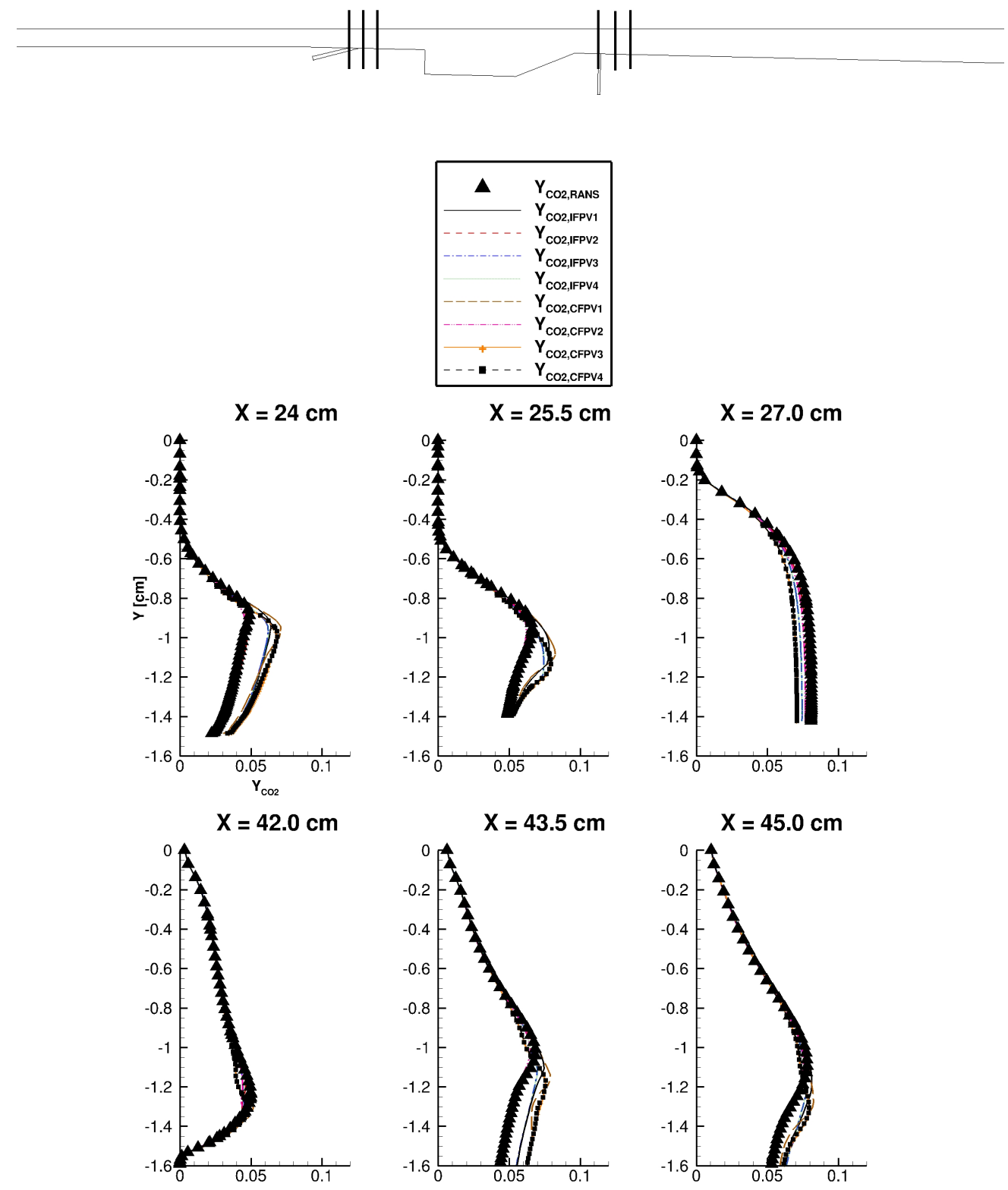

Figure 7. Line plots of $Y$ [cm] versus $Y_{\mathrm{CO}_{2}}$ obtained using the APFM-RANS analysis method and from the baseline RANS simulations of the HDCR combustor at several locations through the primary and secondary injector flames at the injector centerline nearest to the combustor centerline for cases corresponding to IFPV1-IFPV4 and CFPV1CFPV4. Units on each subfigure are the same as those shown on the top-row, left-most subfigure. 

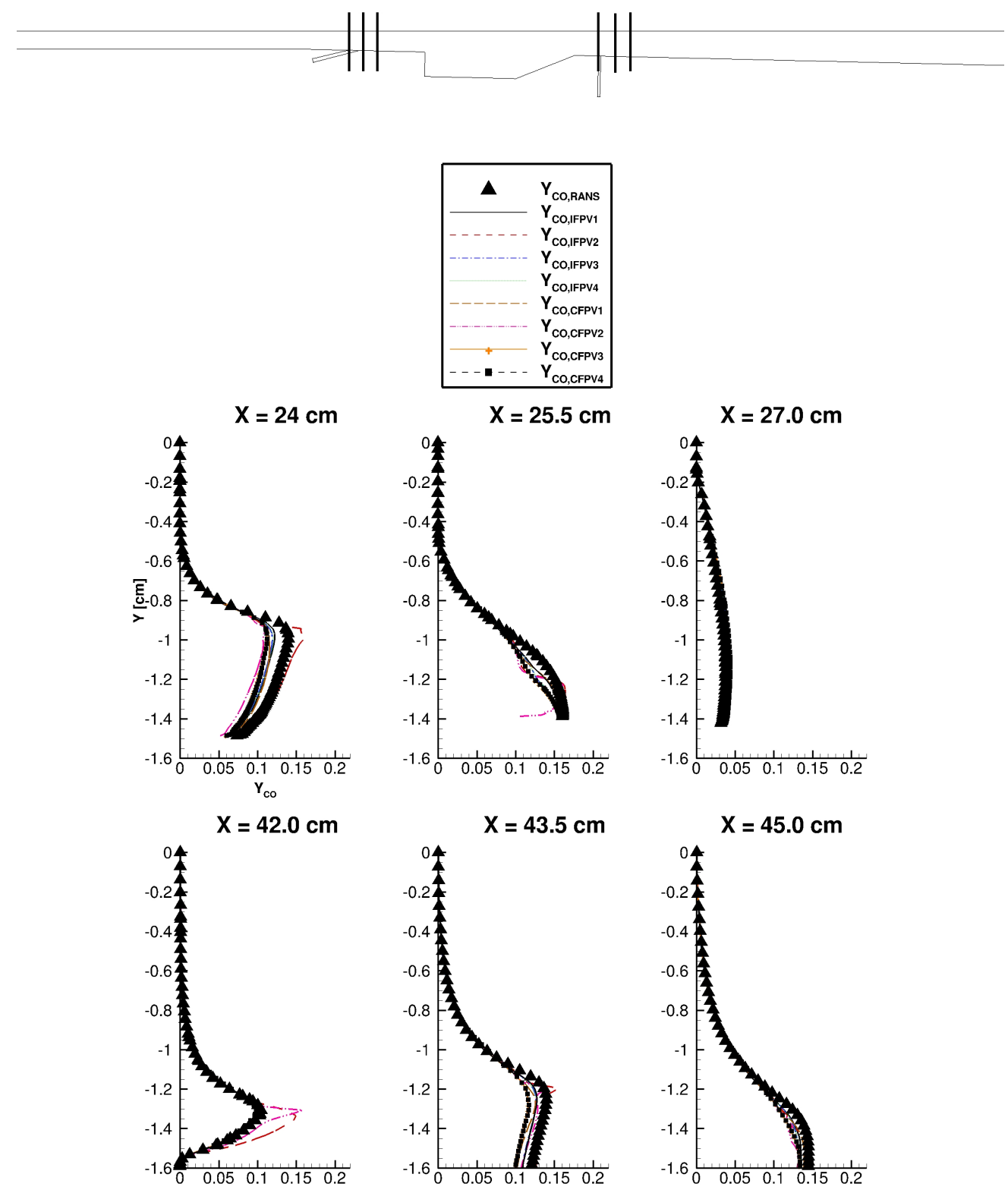

Figure 8. Line plots of $Y$ [cm] versus $Y_{C O}$ obtained using the APFM-RANS analysis method and from the baseline RANS simulations of the HDCR combustor at several locations through the primary and secondary injector flames at the injector centerline nearest to the combustor centerline for cases corresponding to IFPV1-IFPV4 and CFPV1CFPV4. Units on each subfigure are the same as those shown on the top-row, left-most subfigure. 

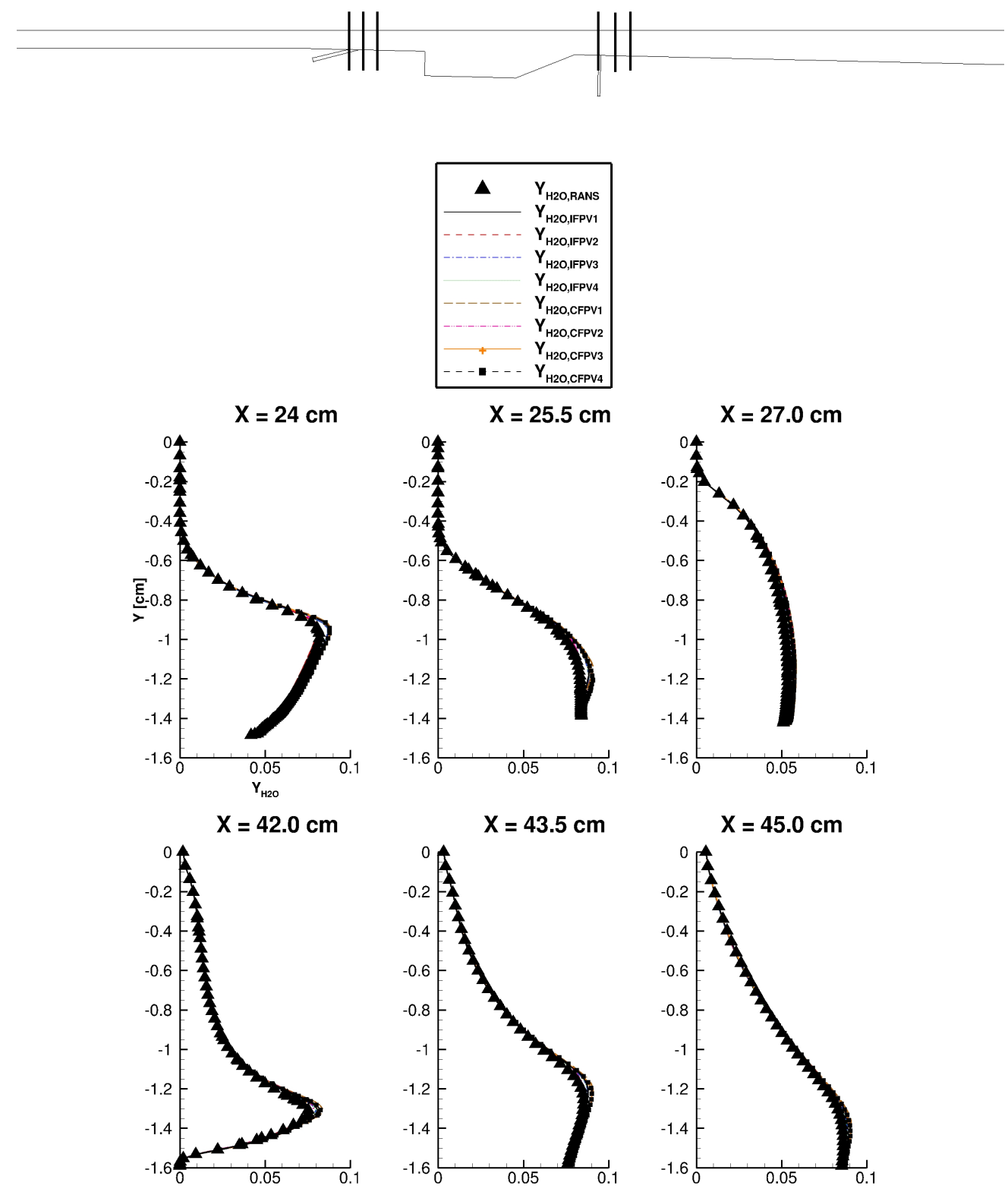

Figure 9. Line plots of $Y$ [cm] versus $Y_{\mathrm{H}_{2} \mathrm{O}}$ obtained using the APFM-RANS analysis method and from the baseline RANS simulations of the HDCR combustor at several locations through the primary and secondary injector flames at the injector centerline nearest to the combustor centerline for cases corresponding to IFPV1-IFPV4 and CFPV1CFPV4. Units on each subfigure are the same as those shown on the top-row, left-most subfigure. 

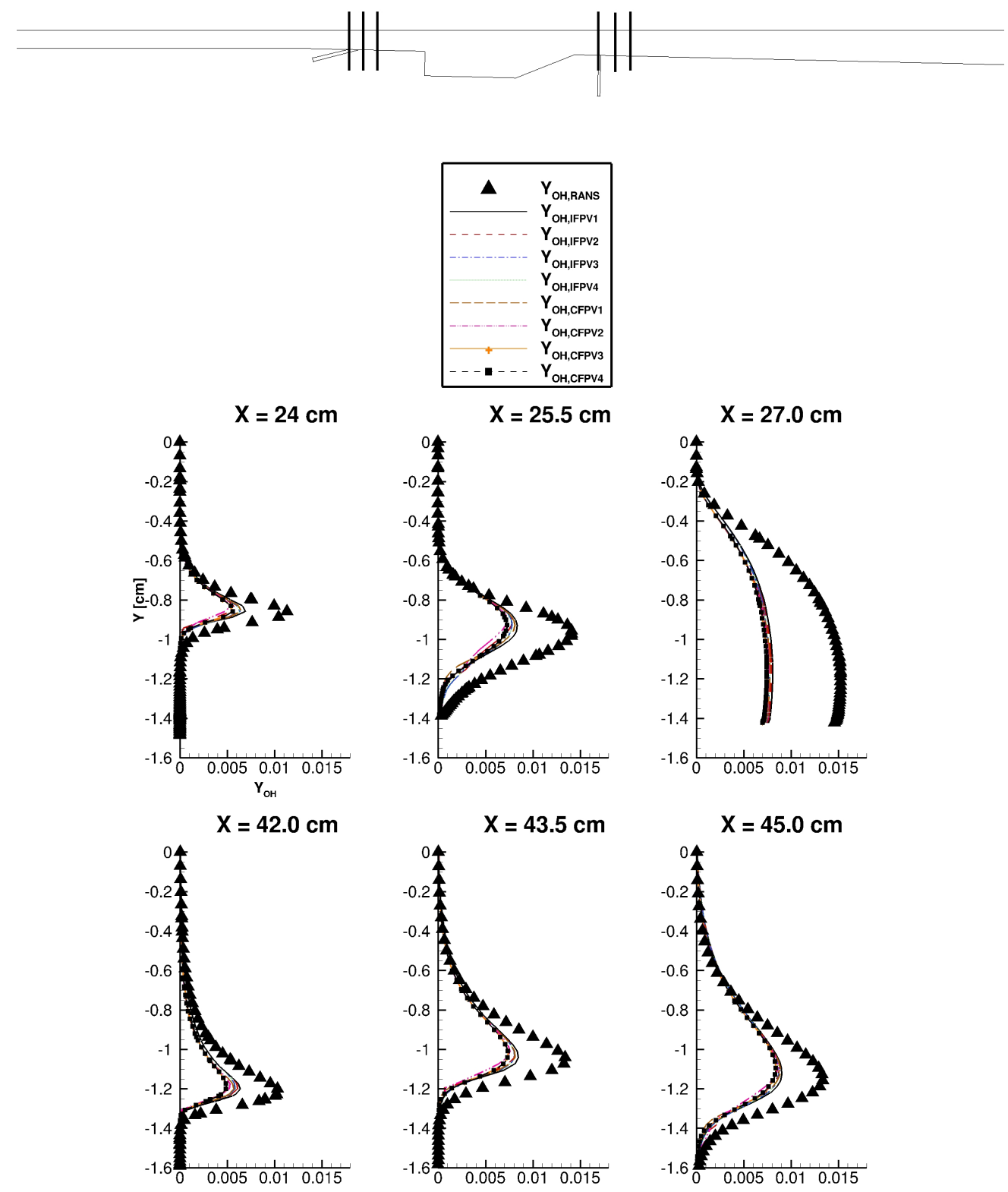

Figure 10. Line plots of $Y[\mathrm{~cm}]$ versus $Y_{O H}$ obtained using the APFM-RANS analysis method and from the baseline RANS simulations of the HDCR combustor at several locations through the primary and secondary injector flames at the injector centerline nearest to the combustor centerline for cases corresponding to IFPV1-IFPV4 and CFPV1CFPV4. Units on each subfigure are the same as those shown on the top-row, left-most subfigure. 
A more detailed comparison can be made by scatter plotting FPV-modeled results against those of the baseline RANS case. These plots are shown for cases IFPV1-IFPV4 and cases CFPV1-CFPV4 in Figs. 11-15 and Figs. 16-20, respectively. In each of these figures, a solid red line of slope one is overlaid on the data as a marker for one-to-one agreement. Deviation from this solid red line indicates the extent of disagreement among the modeled data. Analyzing Figs. 11 and 16 suggests that for IFPV models, the detailed form of $C$ is critical to recovering the $T$ field, whereas for CFPV models, the $T$ field is much less sensitive. Figure 11 shows that the scatter plot corresponding to IFPV4 obtained using Eq. (12) results in a significantly moreaccurate comparison than the other candidate definitions for $C$, whereas in Fig. 16, all four candidates for $C$ result in similarly accurate scatter.

Contrary to the trending in $T$, analyzing results for the selected species mass fractions in Figs. 12-15 and 17-20 suggests the chemical composition exhibits considerably less sensitivity to the inclusion of $p$ as a parameterizing variable in the FPV model. Though, for both the IFPV and CFPV models, the chemical composition exhibits a similarly-high sensitivity to the detailed form of $C$. Also interesting, though perhaps expected, is the trending among the four $C$ candidates, which is not static from one species to the next. For example, in the case of $\mathrm{Y}_{\mathrm{CO}_{2}}$ in Figs. 12 and 17, the results given by Eq. (9) in the top-row, left-most subfigure exhibit the best agreement with the baseline RANS solution; however, for $Y_{\mathrm{H}_{2} \mathrm{O}}$ in Figs. 14 and 19 in the top-row, right-most subfigure, results given by Eq. (10) show the best agreement.
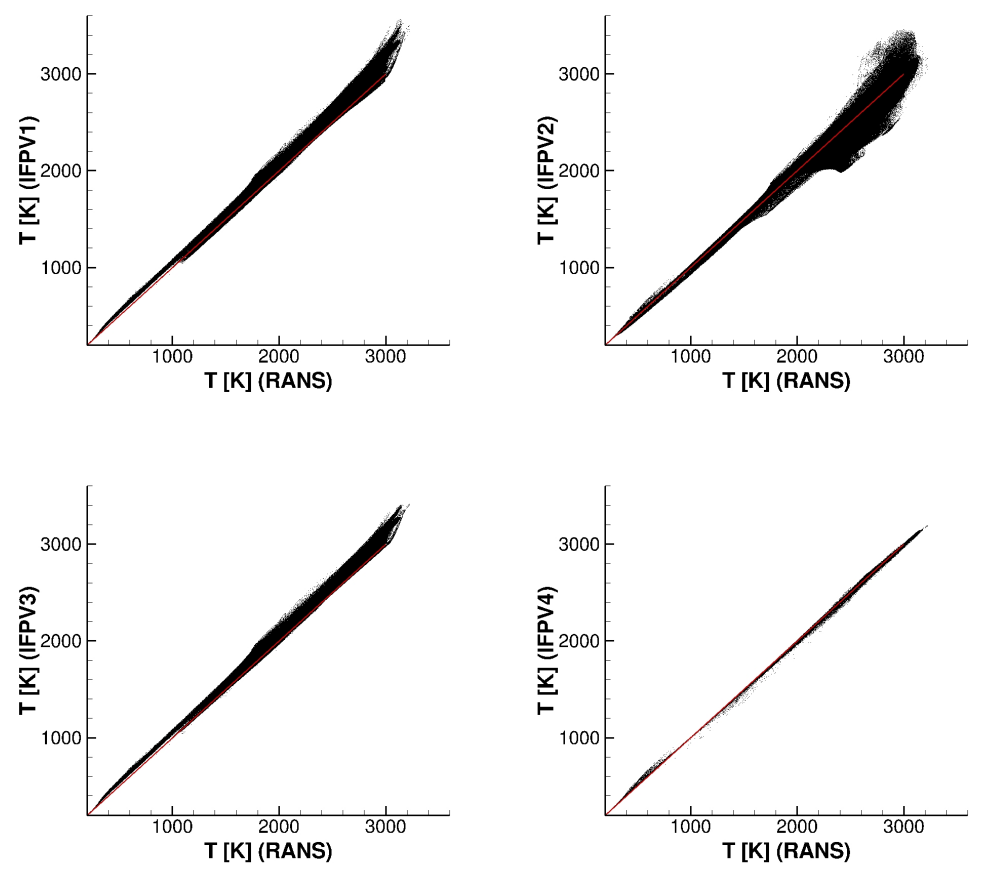

Figure 11. Scatter plots of the static temperature, $T[\mathrm{~K}]$, obtained using the APFM-RANS analysis method versus that from the baseline RANS simulations of the HDCR combustor for the full computational domain for cases IFPV1-IFPV4. The red line denotes a line with slope of one. 

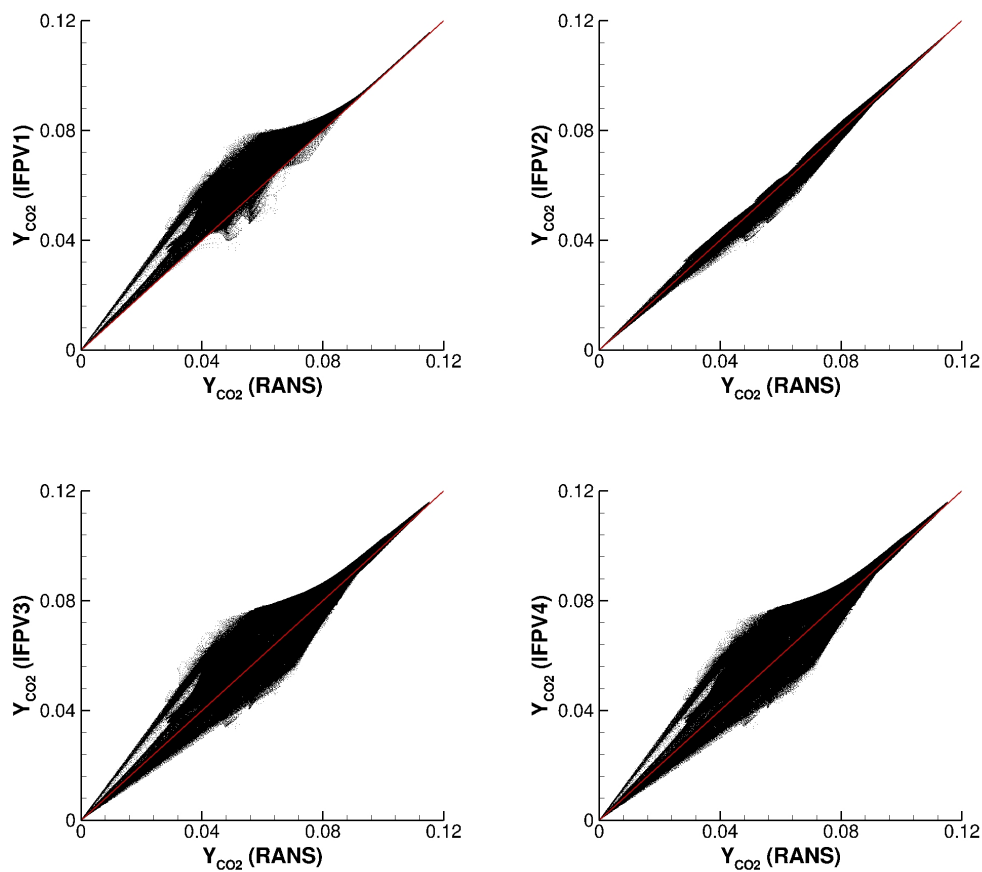

Figure 12. Scatter plots of $Y_{\mathrm{CO}_{2}}$ obtained using the APFM-RANS analysis method versus that from the baseline RANS simulations of the HDCR combustor for the full computational domain for cases IFPV1-IFPV4. The red line denotes a line with slope of one.
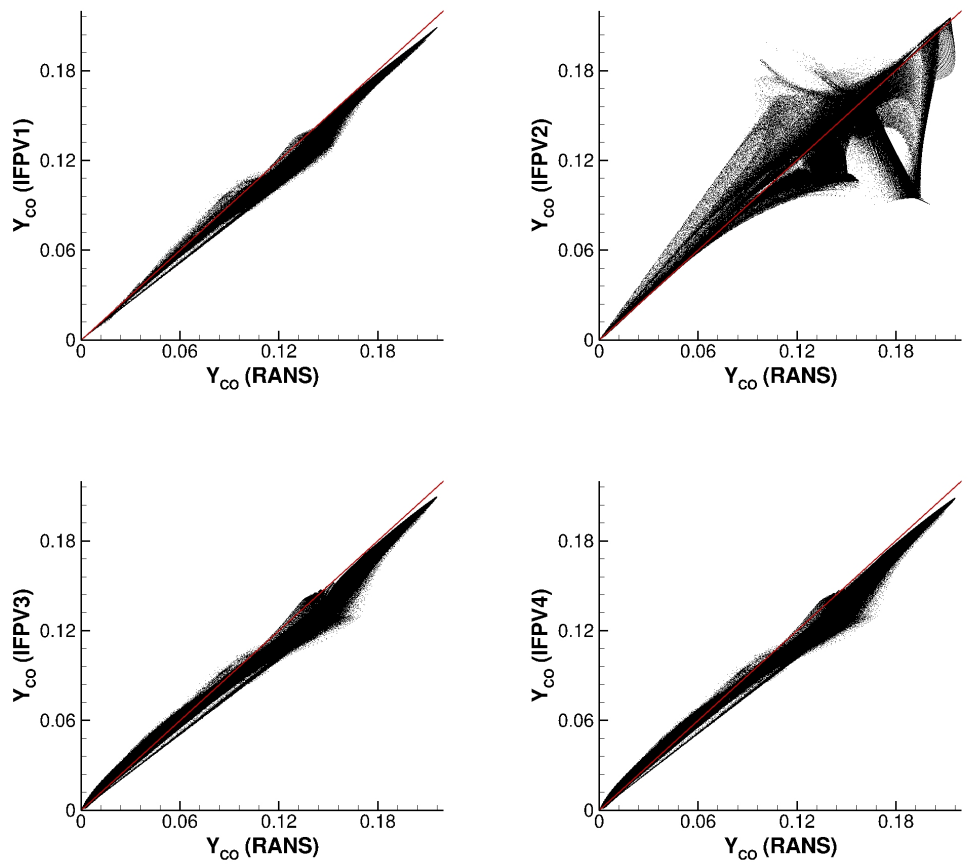

Figure 13. Scatter plots of $Y_{C O}$ obtained using the APFM-RANS analysis method versus that from the baseline RANS simulations of the HDCR combustor for the full computational domain for cases IFPV1-IFPV4. The red line denotes a line with slope of one. 

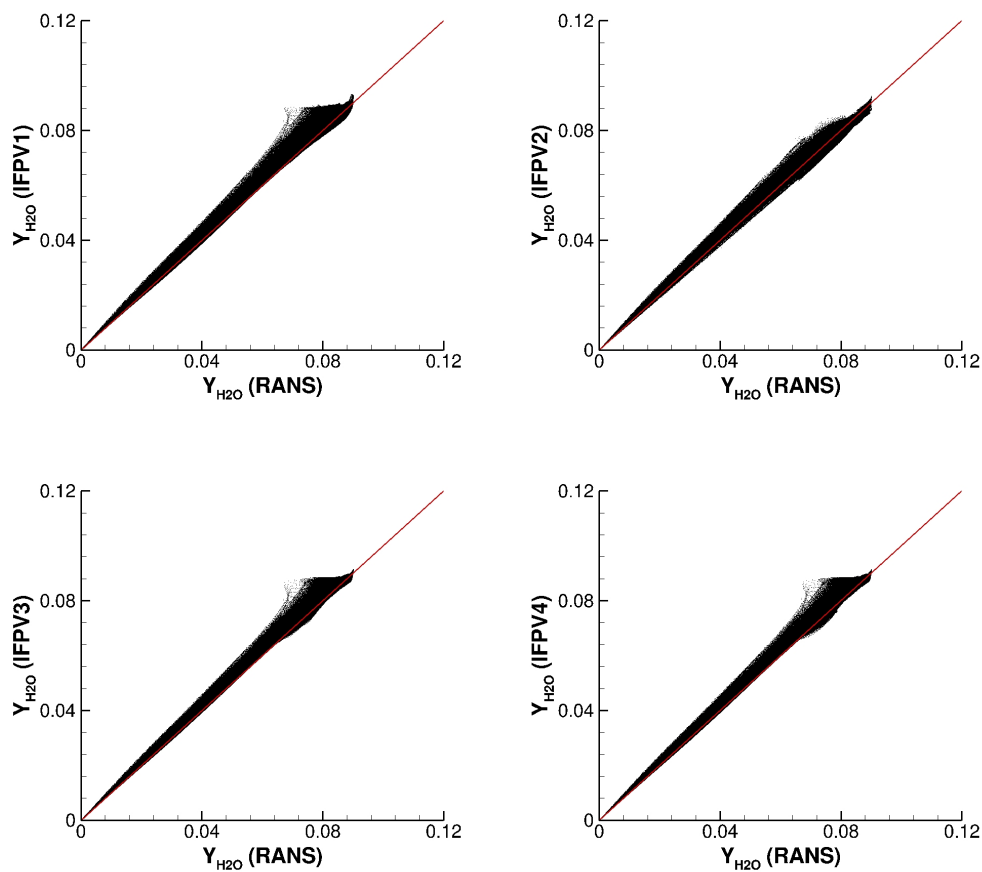

Figure 14. Scatter plots of $Y_{\mathrm{H}_{2} \mathrm{O}}$ obtained using the APFM-RANS analysis method versus that from the baseline RANS simulations of the HDCR combustor for the full computational domain for cases IFPV1-IFPV4. The red line denotes a line with slope of one.
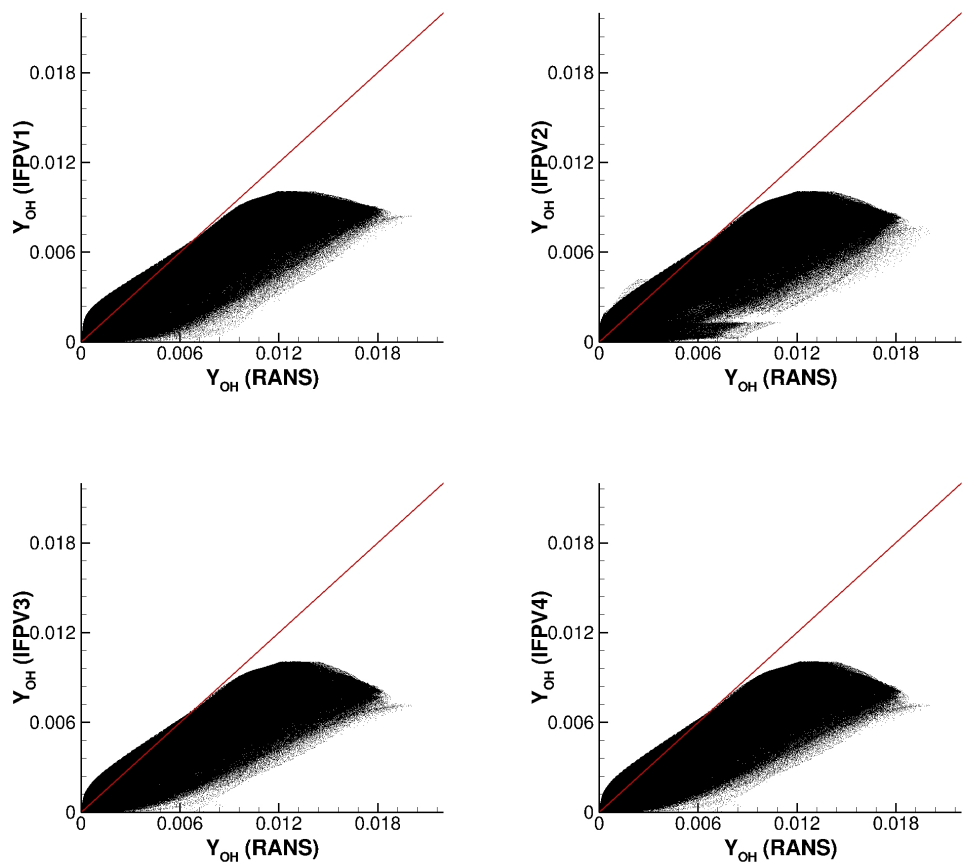

Figure 15. Scatter plots of the temperature, $Y_{O H}$, obtained using the APFM-RANS analysis method versus that from the baseline RANS simulations of the HDCR combustor for the full computational domain for cases IFPV1-IFPV4. The red line denotes a line with slope of one. 

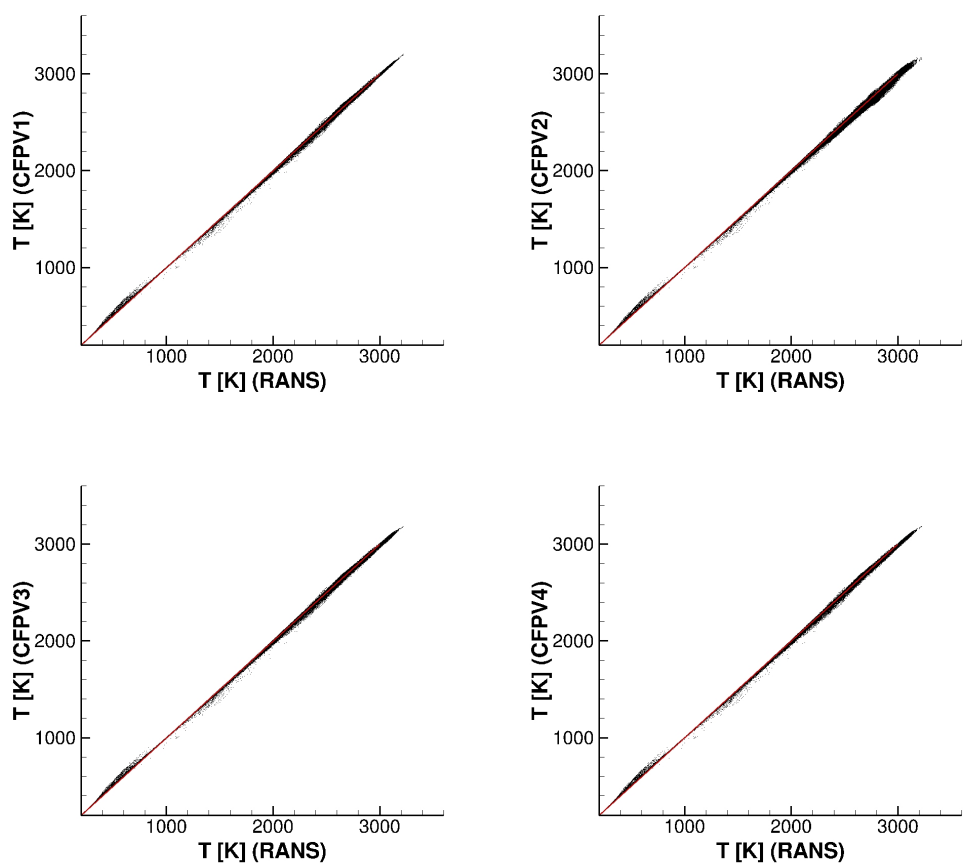

Figure 16. Scatter plots of static temperature, $T[\mathrm{~K}]$, obtained using the APFM-RANS analysis method versus that from the baseline RANS simulations of the HDCR combustor for the full computational domain for cases CFPV1-CFPV4. The red line denotes a line with slope of one.
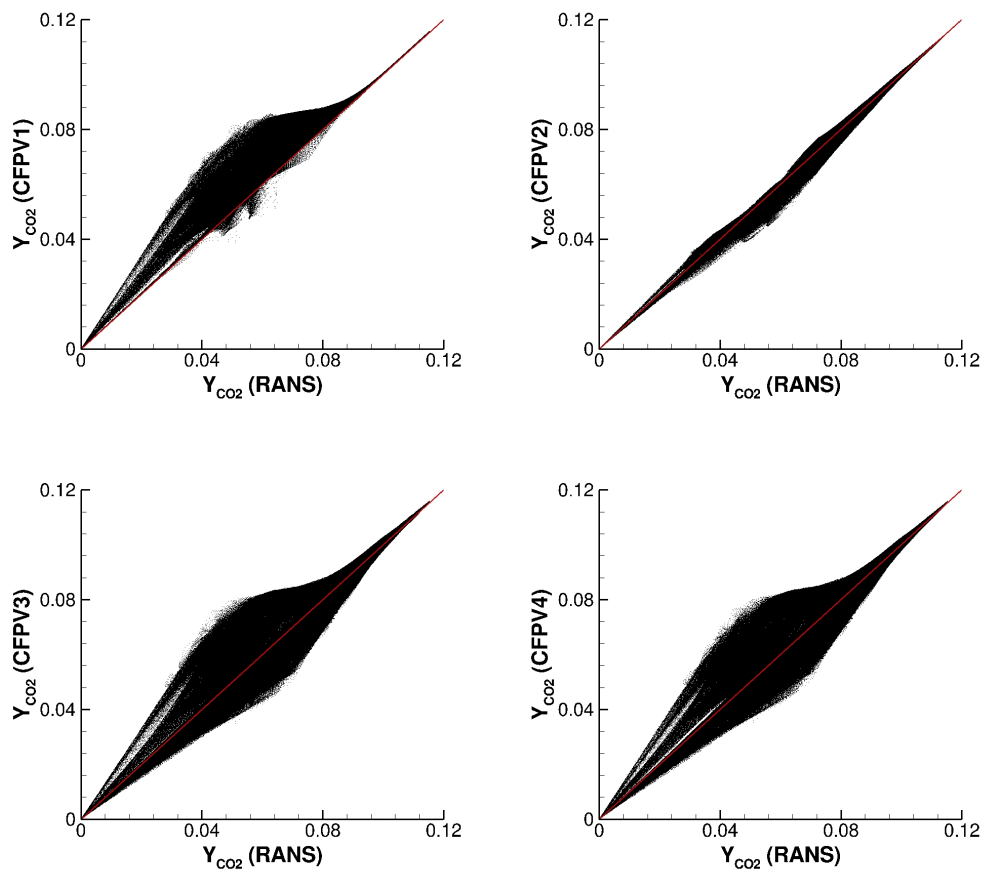

Figure 17. Scatter plots of $Y_{\mathrm{CO}_{2}}$ obtained using the APFM-RANS analysis method versus that from the baseline RANS simulations of the HDCR combustor for the full computational domain for cases CFPV1-CFPV4. The red line denotes a line with slope of one. 

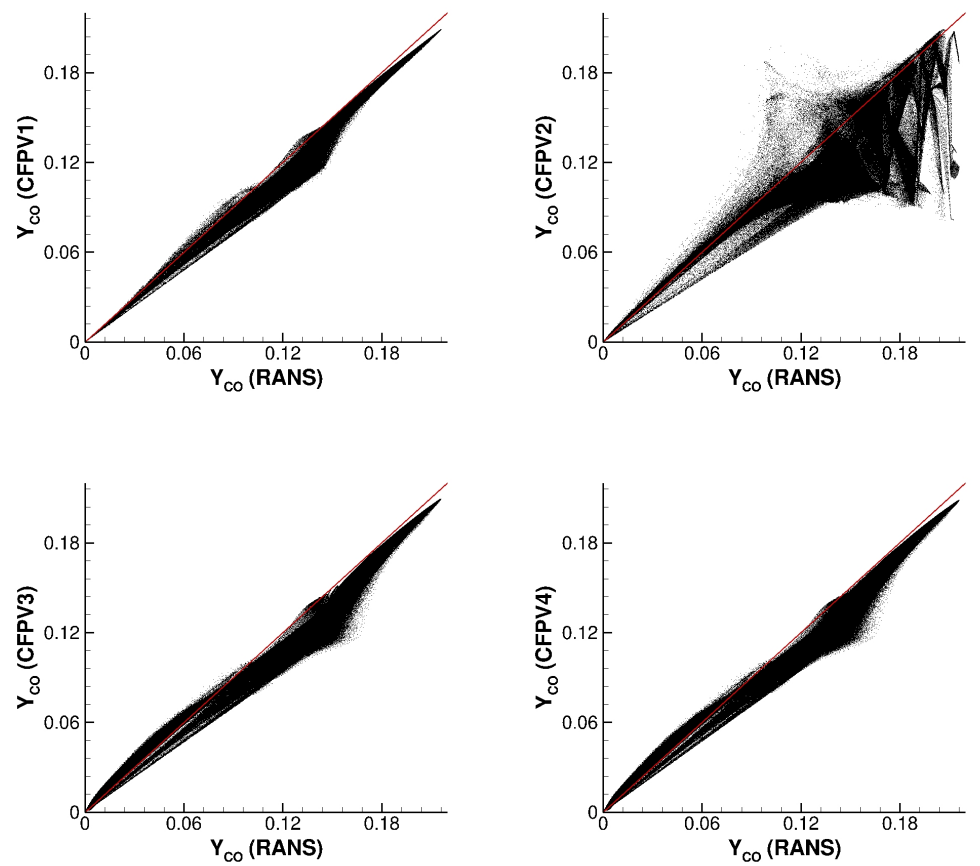

Figure 18. Scatter plots of $Y_{C O}$ obtained using the APFM-RANS analysis method versus that from the baseline RANS simulations of the HDCR combustor for the full computational domain for cases CFPV1-CFPV4. The red line denotes a line with slope of one.
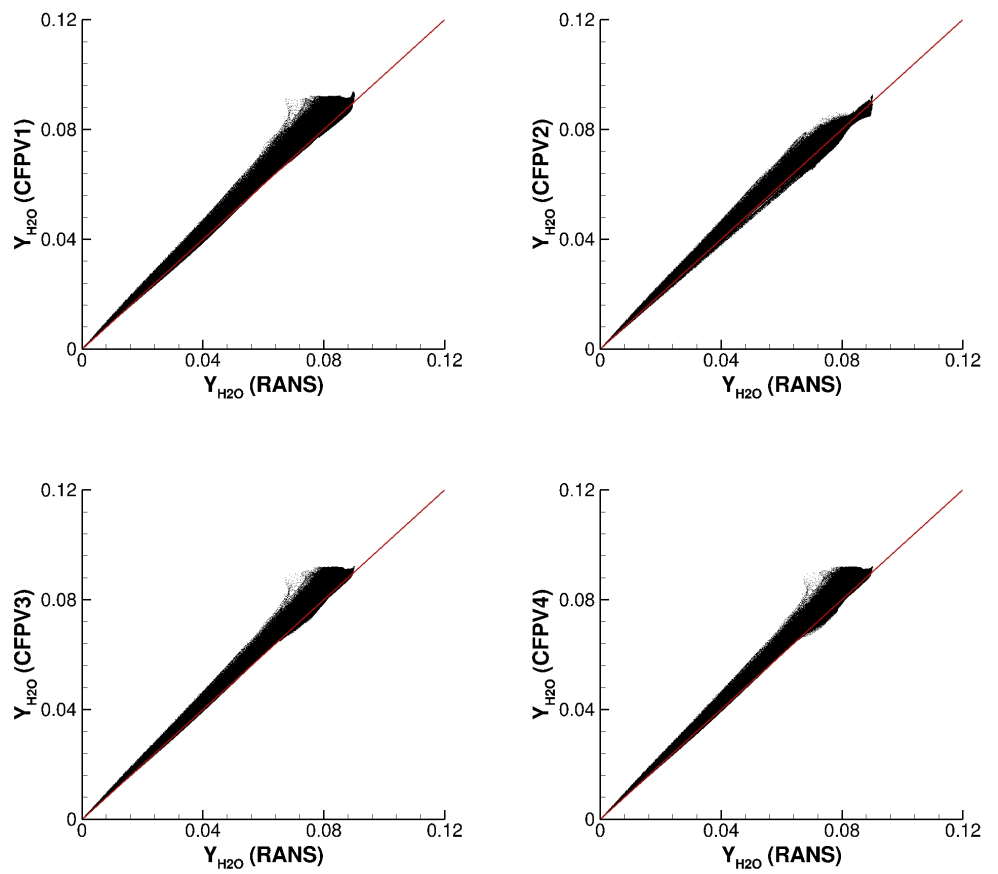

Figure 19. Scatter plots of $Y_{\mathrm{H}_{2} \mathrm{O}}$ obtained using the APFM-RANS analysis method versus that from the baseline RANS simulations of the HDCR combustor for the full computational domain for cases CFPV1-CFPV4. The red line denotes a line with slope of one. 

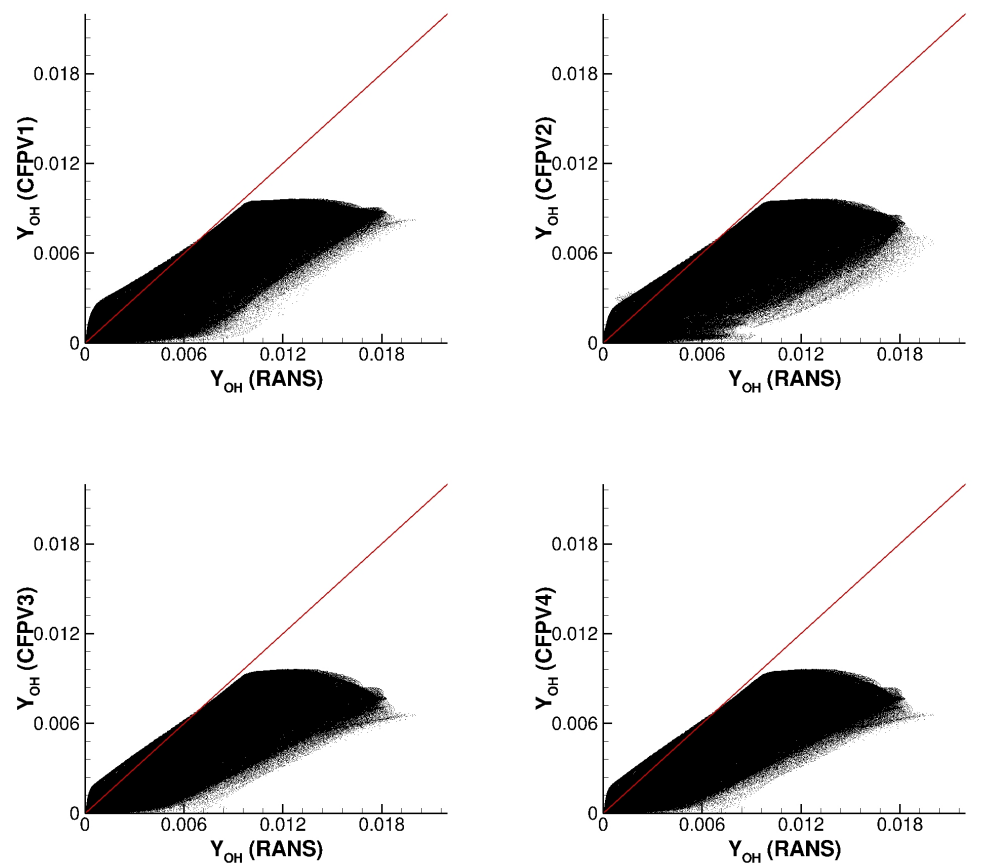

Figure 20. Scatter plots of $Y_{O H}$ obtained using the APFM-RANS analysis method versus that from the baseline RANS simulations of the HDCR combustor for the full computational domain for cases CFPV1-CFPV4. The red line denotes a line with slope of one. 
Based on the comparisons made using Figs. 6-20, several important observations regarding the choice of $C$ and the benefits of using a CFPV model in place of an IFPV model were made. These observations included:

1. When using an IFPV model, the form for $C$ shown in Eq. (12) resulted in the minimal error in the reconstructed $T$ field.

2. Including $p$ as a parameterizing variable via the use of a CFPV model significantly reduced the sensitivity of the reconstructed $T$ field to the detailed form chosen for $C$.

3. The detailed form of $C$ affected each chemical species uniquely.

4. Using a CFPV model in place of the IFPV model resulted in marginal improvements in $Y_{\mathrm{CO}_{2}}, Y_{\mathrm{CO}}$, $Y_{\mathrm{H}_{2} \mathrm{O}}$, and $Y_{\mathrm{OH}}$, while the aggregate effect was an improved prediction for $T$.

Based on the generally good agreement with the baseline RANS solution, the definition for $C$ used throughout the remainder of this study will be the form shown in Eq. (12). One could argue that if $p$ were included as a parameterizing variable in the flamelet model, the definition included in Eq. (9) may be more appropriate based on the slightly better prediction of $Y_{\mathrm{CO}_{2}}$ and $Y_{\mathrm{H}_{2} \mathrm{O}}$, whose production is responsible for significant heat release. However, since both IFPV and CFPV results are considered moving forward, Eq. (12) is used. Note also that the results shown in this section did not consider $\dot{\omega}_{C}$, which is a function of $Y_{\alpha}, p$, and $T$. Results pertaining to $\dot{\omega}_{C}$ are presented in the next section.

\section{III.B. Progress Variable Production Models}

Since the reaction rates used to model the combustion chemistry are strong nonlinear functions of pressure and temperature, the former are expected to be especially sensitive to any modeling errors in the latter. Furthermore, in compressible flows both the pressure and temperature may vary widely across the combustor, not only due to heat release via combustion, but also due to viscous heating, shock waves, and expansion fans. Unfortunately, the detailed variation in these properties is not known a priori; only after a simulation has been performed may the variation of $p$ and $T$ be obtained and sufficiently modeled for an accompanying flamelet table. Herein lies a fundamental problem with FPV models; the temperature values for fuel and oxidizer are needed to set the boundary conditions for the flamelet equations and the value of the pressure of the flamelet itself are not available and cannot be easily computed a priori. Further, $\dot{\omega}_{C}$ is generally used directly from the flamelet table-meaning that the $p$ and $T$ used in the calculation of $\dot{\omega}_{C}$ are those corresponding to the solution of the flamelet equations and not those of the local compressible flowfield. For high-speed, compressible, reacting flows, the difference between the temperature and pressure embedded in the flamelet table and that of the local flow field are typically significantly different, and when this difference is considered without variation in $p$ and the strong nonlinearity of $\dot{\omega}_{C}$, it is clear that for FPV models the tabulated $\dot{\omega}_{C}$ contains considerable error. In this section, several possible methods for reducing this error in $\dot{\omega}_{C}$ are evaluated.

The most direct way to account for pressure dependencies on the combustion in an FPV model is to include pressure as a parameterizing variable, as discussed in previous sections, and solve the flamelet equations over a range of pressure. For cases in which model efficiency is paramount, Terrapon et al. ${ }^{4}$ used a scaling model to account for pressure effects on the $\dot{\omega}_{C}$. In their approach, instead of tabulating flamelet solutions over a range of pressure levels, an IFPV approach is used with a slight modification to the $\dot{\omega}_{C}$ term. When $\dot{\omega}_{C}$ is retrieved from the table, it is scaled by the local pressure according to Eq. (13), where $p_{0}$ and $\dot{\omega}_{C_{0}}$ are the static pressure and net production rate of $C$, respectively, corresponding to the flamelet solution.

$$
\dot{\omega}_{C}=\frac{p^{2}}{p_{0}^{2}} \dot{\omega}_{C_{0}}
$$

The squared scaling on pressure results from an assumed predominance of bimolecular reactions. However, this scaling only attempts to correct for pressure effects on the $\dot{\omega}_{C}$, when in reality the entirety of the tabulated data is generally a function of pressure, including $Y_{\alpha}, \frac{\partial Y_{\alpha}}{\partial Z}$, etc. These effects on the additional tabulated data are captured only for FPV models that explicitly parameterize the flamelet state-space over pressure. Furthermore, both fuel and oxidizer temperature vary throughout the flowfield. Therefore, the values of the temperature, species mass fractions, and reaction rates recovered from the flamelet table may 
contain large errors. In general, not much can be done regarding the species mass fractions, but some corrections may be introduced for both the temperature and reaction rates. In this work, we ignore the temperature from the flamelet table and instead choose to use an iterative procedure to obtain it from the transported energy variable. For the reaction rates, we consider several correction strategies that aim to improve the values.

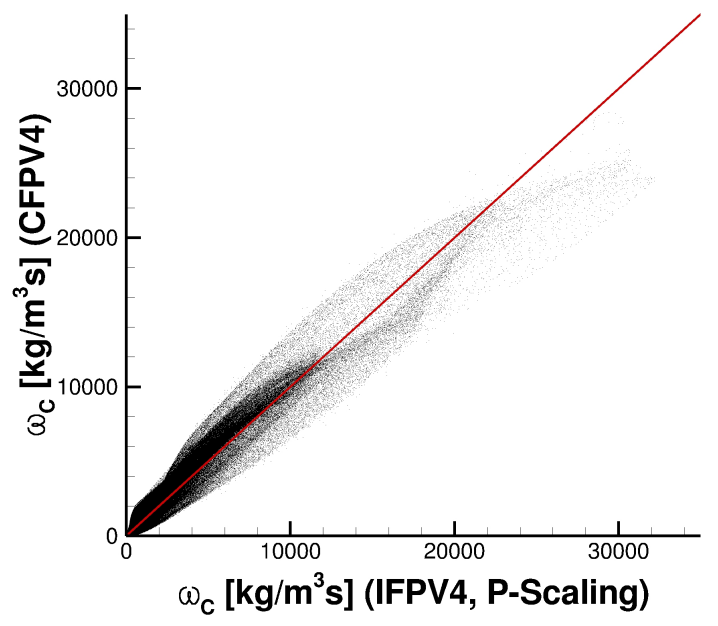

Figure 21. Scatter plot of net production rate of $C, \dot{\omega}_{C}\left[\mathrm{~kg} / \mathrm{m}^{3} \mathrm{~s}\right]$, calculated using the APFM-RANS analysis method for case IFPV4 with the pressure-scaling model in Eq. (12) as compared to that of case CFPV4, for which $\dot{\omega}_{C}$ is parameterized explicitly by pressure. The red line denotes a line with slope of one.

To gauge the accuracy of the pressure-scaling approach, $\dot{\omega}_{C}$ for case IFPV4 was recomputed using Eq. (13) and was compared to that of case CFPV4. This comparison is shown in Fig. 21, which demonstrates the pressure-scaled $\dot{\omega}_{C}$ agrees reasonably well with the results of case CFPV4, in which the flamelet solutions are explicitly solved at several pressure levels. The greater disagreement at higher values of $\dot{\omega}_{C}$ may be a result of trimolecular reactions reflected in case CFPV4 but omitted from the pressure-scaling model with case IFPV4. If this were true, using a higher exponent in Eq. (13) may help to reduce this discrepancy. These results were also compared to the baseline RANS solution in flame regions around each injector in Fig. 22, and scatter plot comparisons of these results to those of the baseline RANS solution are shown for the entire combustor in Fig. 23. Note that a third scaling approach based on pressure and temperature is included in Fig. 22, which will be discussed later.

In Fig. 22, the results indicate that in flame regions both approaches agree similarly well with the baseline RANS data, thereby suggesting both approaches offer similarly accurate $\dot{\omega}_{C}$ values. However, note that while the $\dot{\omega}_{C}$ may agree reasonably well for both pressure-scaling approaches, pressure effects are not captured for any other table data using this approach. Specifically, the agreement with the RANS data for the $Y_{\alpha}, T$, and $\frac{\partial Y_{\alpha}}{\partial Z}$ are identical to that of the IFPV model results. Only by using pressure as a parameterizing variable in the flamelet table may the effects of pressure be reflected in these additional terms.

An additional correction on the tabulated $\dot{\omega}_{C}$ involves correcting for the temperature effect on the reaction rates in a similar scaling method as was used for pressure. One such scaling method is shown below in Eq. (14), where $\rho_{0}$ and $T_{0}$ are the density and temperature corresponding to the flamelet solution, and $a_{p}$ and $T_{a}$ are modeling parameters representative of the reaction kinetics and activation energy, respectively. ${ }^{39}$

$$
\dot{\omega}_{C}=\left(\frac{\rho}{\rho_{0}}\right)^{a_{p}} \exp \left[-T_{a}\left(\frac{1}{T}-\frac{1}{T_{0}}\right)\right] \dot{\omega}_{C_{0}}
$$

This expression employs a scaling for $\dot{\omega}_{C}$ using an Arrhenius-inspired form. The 0 subscript indicates a parameter from a flamelet solution, and $a_{p}$ and $T_{a}$ are computed in a pre-processing step. Most of the kinetic steps involved with hydrocarbon chemistry are bimolecular, so a value of 2 was used for $a_{p}$, as suggested by Saghafian et al. ${ }^{39} \mathrm{~A}$ value of $10,000 \mathrm{~K}$ was used for $T_{a}$ as a best-fit value based a trial-and-error approach. Eq. (14) can be recast using the equation of state to better illustrate the pressure and temperature scalings, as is done below in Eq. (15). 

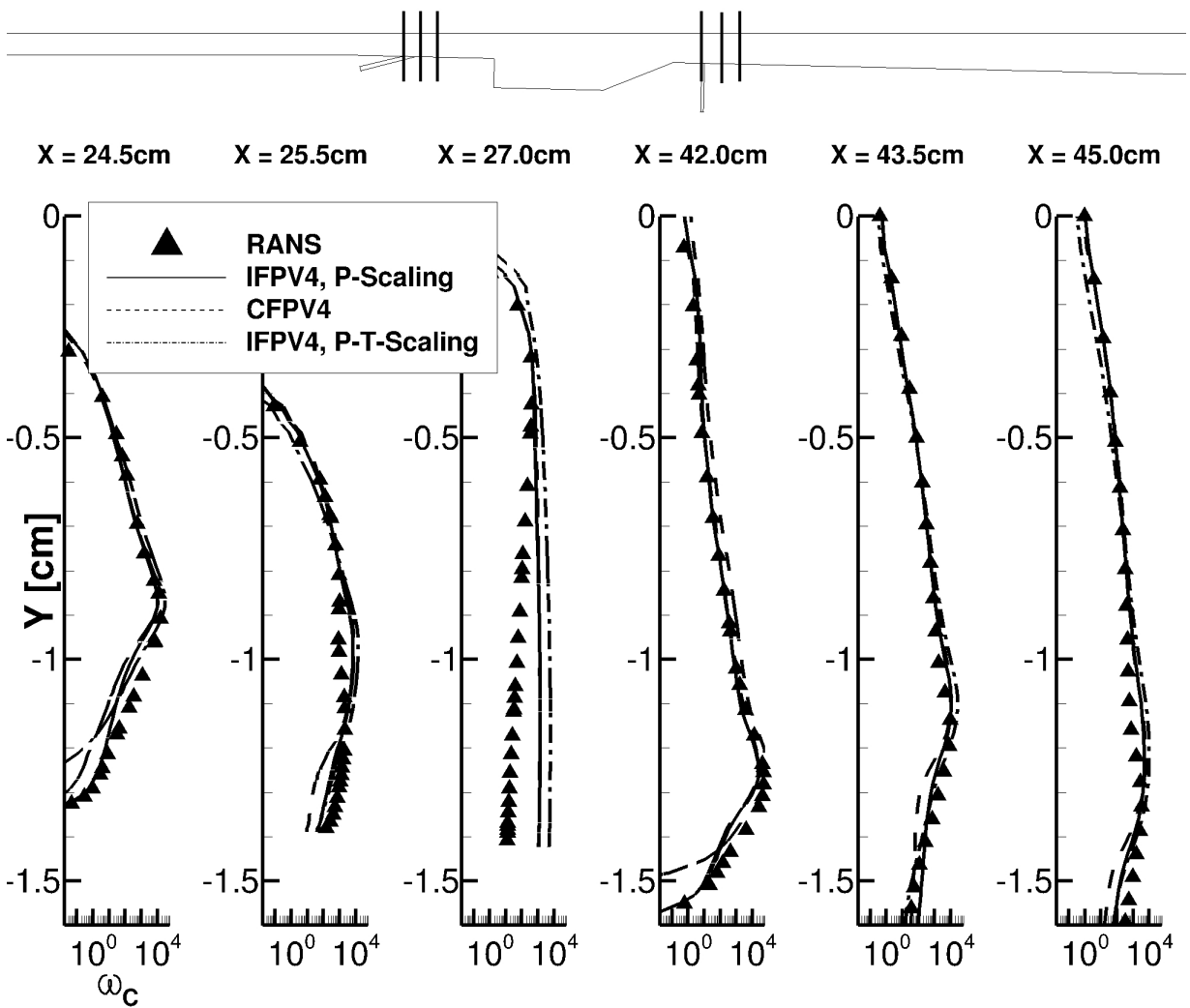

Figure 22. Line plot of $Y$ [cm] versus net production rate of $C, \dot{\omega}_{C}\left[\mathrm{~kg} / \mathrm{m}^{3} \mathrm{~s}\right]$, calculated using the APFM-RANS analysis method for case IFPV4 with the use of Eq. (13), case CFPV4, and case IFPV4 with the use of Eq. (15) to baseline RANS data at selected locations through the primary and secondary injectors spanning the flame regions. Locations of the sampled data planes are shown on the combustor geometry cross-section. 


$$
\dot{\omega}_{C}=\left(\frac{p}{p_{0}}\right)^{a_{p}}\left(\frac{T_{0}}{T}\right)^{a_{p}} \exp \left[-T_{a}\left(\frac{1}{T}-\frac{1}{T_{0}}\right)\right] \dot{\omega}_{C_{0}}
$$

Thus, Eq. (14) consists of both a pressure- and temperature-scaling, where the pressure-scaling is identical to that of Eq. (13) and the temperature-scaling takes on the form shown in Eq. (15). Using this approach in practice to model both pressure and temperature effects on $\dot{\omega}_{C}$ can be done by either using Eq. (15) directly with an IFPV model or by using a pressure-dependent FPV model, such as that used in cases CFPV1-CFPV4, together with the temperature-scaling only shown in Eq. (16).

$$
\dot{\omega}_{C}=\left(\frac{T_{0}}{T}\right)^{a_{p}} \exp \left[-T_{a}\left(\frac{1}{T}-\frac{1}{T_{0}}\right)\right] \dot{\omega}_{C_{0}}
$$

To demonstrate the performance of the above corrections, four FPV solutions are compared to the baseline RANS solution in Fig. 23. These solutions included: the results of case IFPV4, the pressure-scaled results of case IFPV4 using Eq. (13), the pressure-temperature-scaled results of case IFPV4 using Eq. (15), and the temperature-scaled results of case CFPV4 using Eq. (16). These comparisons demonstrate the strong influence of both pressure and temperature corrections on the value of $\dot{\omega}_{C}$, and for the current study, both pressure- and temperature-scalings will be used to recover the $\dot{\omega}_{C}$. Similarly to Fig. 21, the difference between using scaling models with case IFPV4 versus using the temperature-scaling in Eq. (16) with case CFPV4 is small for $\dot{\omega}_{C}$; however, note that the scaling models discussed here only apply to $\dot{\omega}_{C}$ and that any pressure effects for the other flamelet table terms are neglected by using a scaling model.
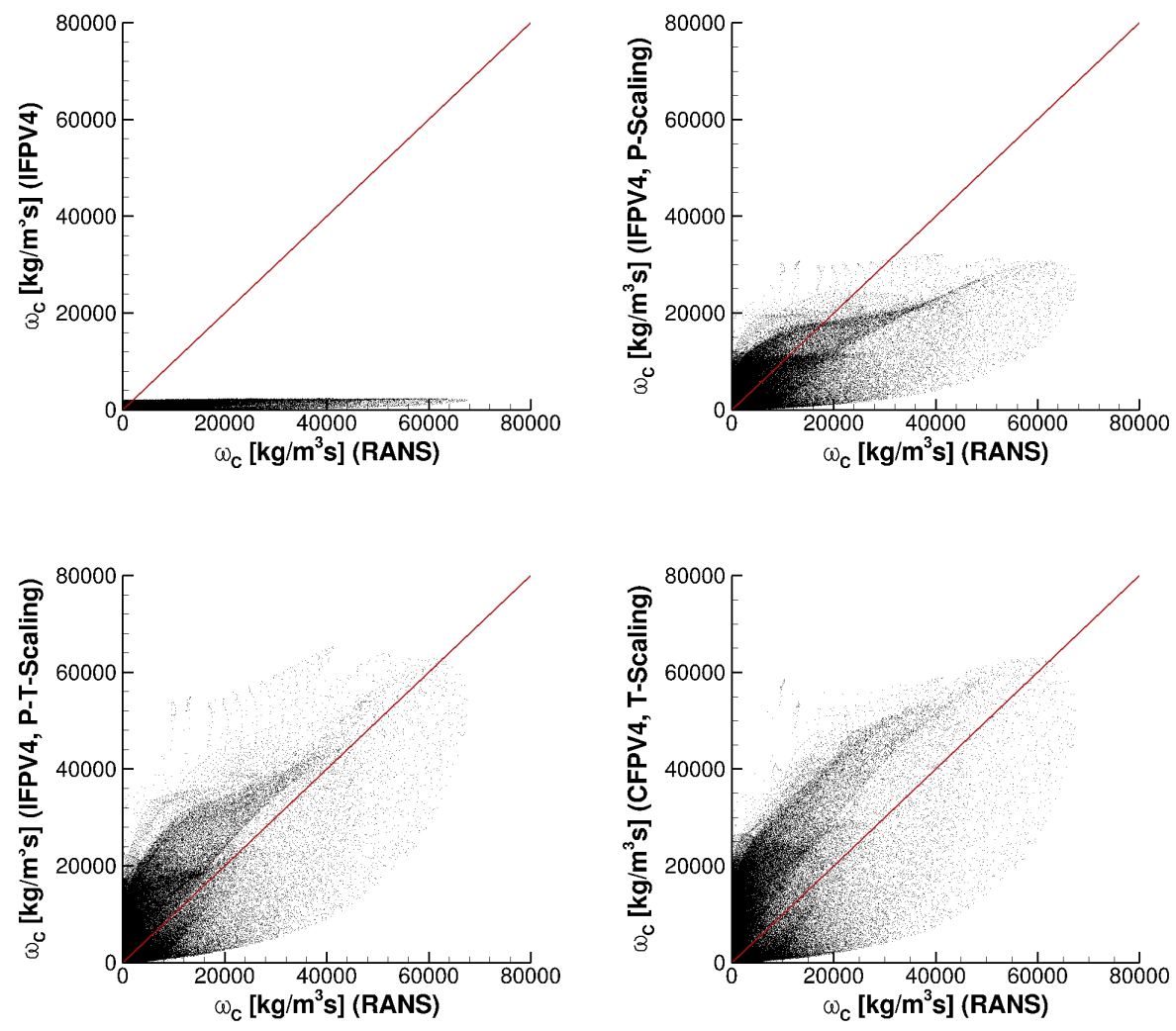

Figure 23. Scatter plot comparisons of APFM-RANS analysis results and the baseline RANS data for net production rate of $C, \dot{\omega}_{C}\left[\mathrm{~kg} / \mathrm{m}^{3} \mathrm{~s}\right]$, for the entire computational domain for cases: (top-left) IFPV4, (top-right) IFPV4 with the pressure-scaling model shown in Eq. (13), (bottom-left) IFPV4 with the pressure- and temperature-scaling models shown in of Eq. (14), and (bottom-right) CFPV4 with the pressure-scaling model shown in Eq. (16). 


\section{III.C. FPV Model Coupling}

In every FPV model implementation, the flamelet table and associated thermochemical state-space must be coupled to the governing transport equations. This coupling is generally performed using Eqs. (1), (2), and (3) and by retrieving $Y_{\alpha}$ and $\dot{\omega}_{C}$ from the flamelet table. In this work, however, alternative approaches for calculating the energy interdiffusion term in Eq. (3) are considered. Equation (17) shows the energy interdiffusion term that appears on the right hand side of the total energy transport equation. In this equation, $\rho$ and $D$ denote the density and mixture average diffusivity, respectively. Similar terms that require coupling exist in other forms of the energy equation, as well. Several approaches for calculating this term are considered in this section.

Direct calculation of the energy interdiffusion term is straight forward when transport equations for $Y_{\alpha}$ are solved. However, for FPV model implementations, data from the flamelet table are required to compute this term, since only transport equations for $Z$ and $C$ are solved. Though the $Y_{\alpha}$ could be taken directly from the flamelet table at runtime to calculate this term, this approach would require numerically differentiating $Y_{\alpha}$ with respect to each space dimension within the solver, which would require significant computational resources. Alternatively, this term can be recast using the chain rule on the flamelet manifold parameterizing variables. Performing this decomposition would yield derivatives of $Y_{\alpha}$ with respect to each of the manifold dimensions and would require the tabulation of each in the flamelet library. In order to maintain a reasonably-compact flamelet library, only contributions in $Z$ space are considered for the current model, for which only $\frac{\partial Y_{\alpha}}{\partial Z}$ will require storage. A third option for calculating this term is to simply tabulate the entire summation appearing on the left-hand-side of Eq. (17) and make it available directly from the flamelet solutions. The three choices for computing the energy interdiffusion term, denoted as $\beta$, are shown below in Eq. (17), where data retrieved from the flamelet table are colored red.

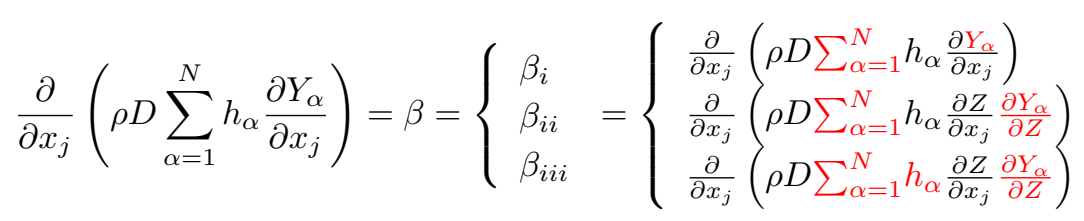

Since option $\beta_{i}$ requires $3 N$ numerical differentiations of $Y_{\alpha}$ for a three-dimensional problem, this approach was ruled out on account of processing requirements. Options $\beta_{i i}$ and $\beta_{i i i}$ differ only in their calculation of $h_{\alpha}$ and their tabulation requirements. For $\beta_{i i}$, the transported temperature is used to calculate $h_{\alpha}$,

and $\frac{\partial Y_{\alpha}}{\partial Z}$ must be stored for all $N$ species. Alternatively, for $\beta_{i i i}$, the local flamelet temperature is used to compute $h_{\alpha}$, which then allows for the storage and retrieval of a single term from the table.

In order to determine whether the presumed increase in accuracy of $\beta_{i i}$ outweighed the table consolidation permitted by $\beta_{i i i}$, both of these calculations were performed using the APFM-RANS analysis method. Scatter plots of the two terms against the baseline RANS data demonstrates the level of modeling error associated with both, as shown in Fig. 24 for $\beta_{i i}$ and Fig. 25 for $\beta_{i i i}$. As seen by comparing these two figures, the additional information required to calculate $\beta_{i i}$ generally results in a more accurate prediction for the energy interdiffusion, as compared to using $\beta_{i i i}$. In particular, $\beta_{i i i}$ diverges from the baseline RANS data substantially at large negative values, while $\beta_{i i}$ closely tracks the baseline RANS data through the same region. The variability of $\beta_{i i}$ around the red line with a slope of one also appears to be less than that of $\beta_{i i i}$. Based on these observations, the $\beta_{i i}$ formulation is chosen for the current analysis; however, for cases employing significantly larger reaction mechanisms for construction of the flamelet table, the error in using $\beta_{i i i}$ may be justifiable for the table consolidation offered by this term.

\section{III.D. CFPVX Model}

The final objective of the current study was to demonstrate the validity of the proposed CFPVX model using the APFM-RANS analysis method. In this approach, the three-dimensional CFPV model is extended to include a fourth dimension that accounts for the effects of variability in flamelet oxidizer temperature, $T_{o x}$. Since a transport equation cannot be solved for $T_{o x}$ easily, the table is mapped to the $Z$ - $C$ - $p$ - $h$ fourdimensional state-space. This formulation is similar to previous attempts ${ }^{40,41}$ at including the effects of non-adiabatic heat transfer on the flamelet manifold through the inclusion of $h$ as an additional dimension. The values of $T_{o x}$ are obtained from the baseline RANS data and converted to a local value of the mixture enthalpy, $h$. APFM-RANS analyses for each of the four progress variable definitions shown in Eqs. (9)-(12) were performed in cases CFPVX1-CFPVX4, for which analysis details are summarized in Table 1. However, 


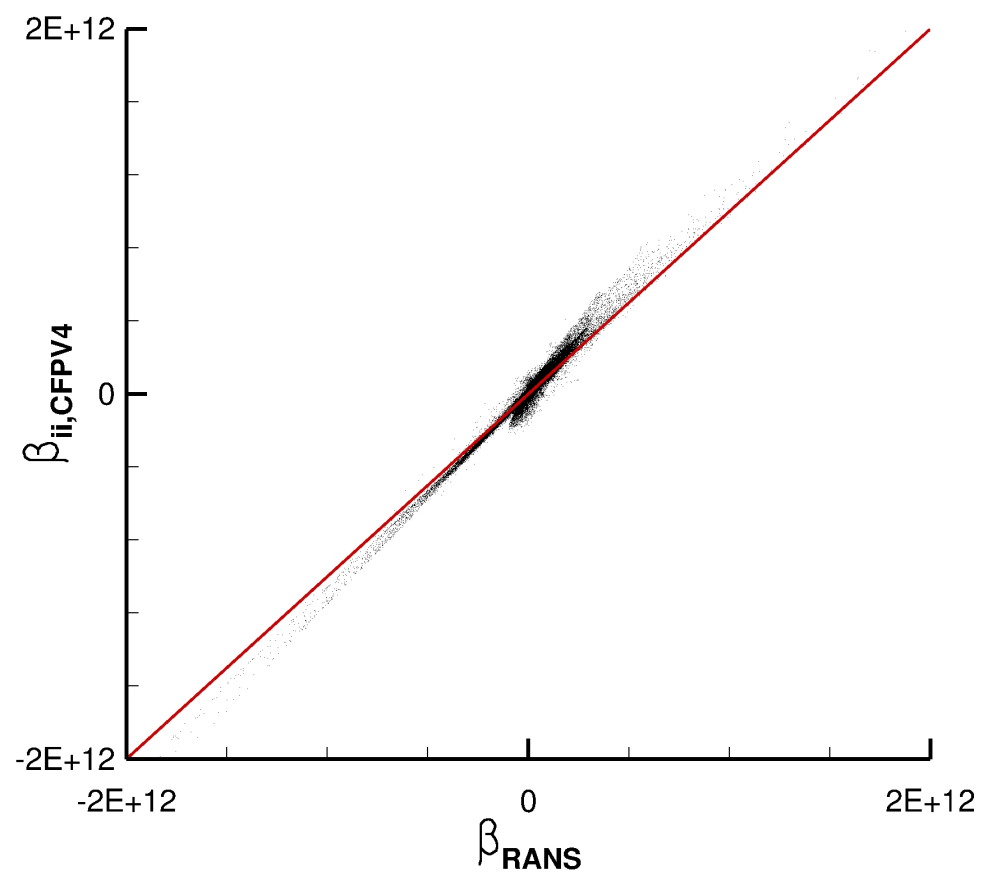

Figure 24. Scatter plot of energy interdiffusion, $\beta$, using the $\beta_{i i}$ formulation for case CFPV4 compared to the baseline RANS data. The red line denotes a line with slope of one.

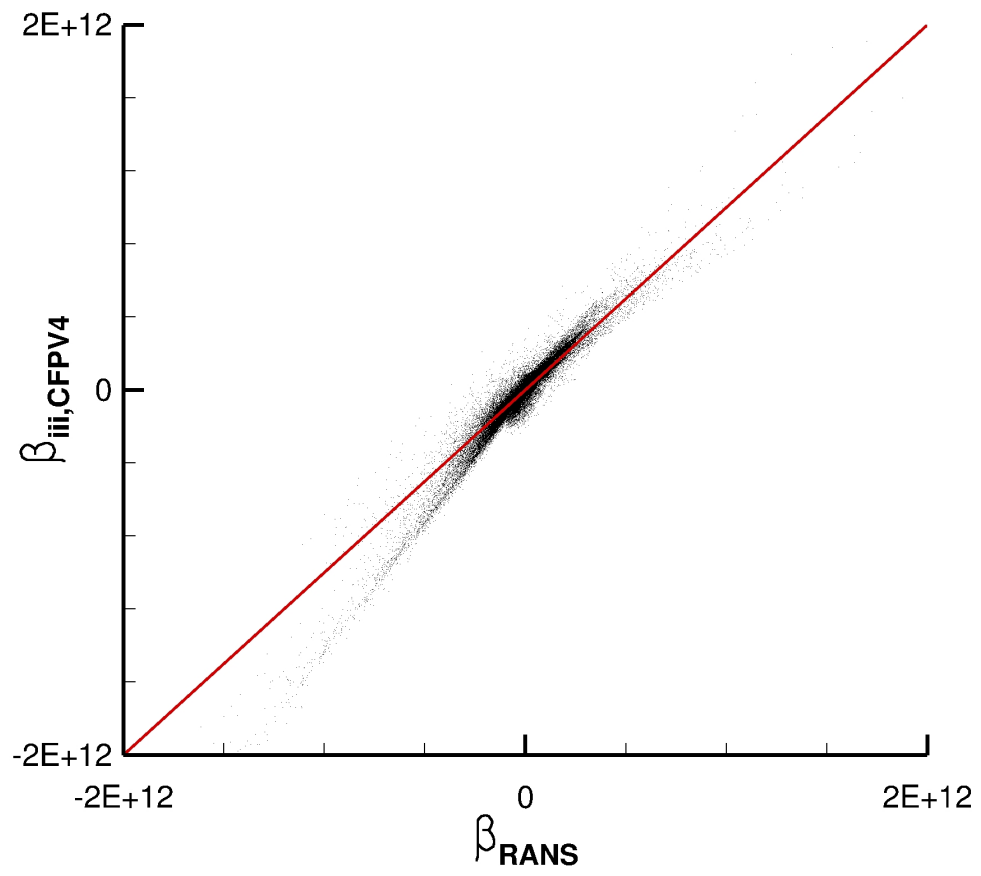

Figure 25. Scatter plot of energy interdiffusion, $\beta$, using the $\beta_{i i i}$ formulation for case CFPV4 compared to the baseline RANS data. The red line denotes a line with slope of one. 
based on the observations in preceding sections, only results from case CFPVX4 are presented in the current section and are compared to the baseline RANS data.

Figure 26 shows how the mixture enthalpy changes within the $Z, C, T_{o x}$ flamelet manifold. The manifold data points are colored by $h$, which shows that the $T_{o x}$-to- $h$ mapping is reasonably monotonic across the entire manifold. There exists some small level of multivaluedness around stoichiometry and in regions nearing $Z=1$; however, this effect is small, as will be discussed later.

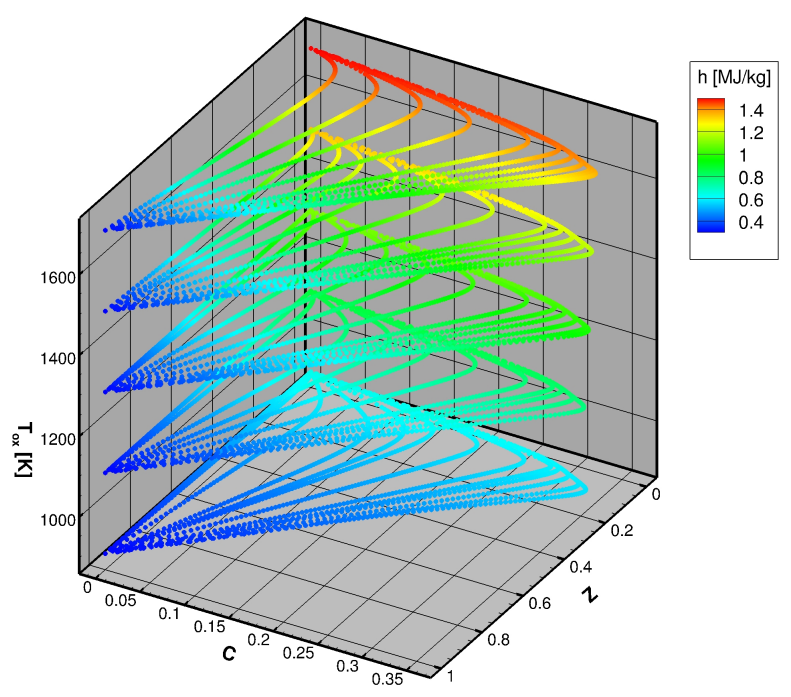

Figure 26. The flamelet manifold embedded in table FT3 presented in mixture fraction-progress variable-oxidizer temperature $\left(Z-C-T_{o x}\right)$ space where points are colored by mixture enthalpy, $h$, to demonstrate the monotonic nature of the $T_{o x}-h$ mapping.

Similar to previous sections, results for the CFPVX4 model are presented in the form of line plots and scatter plots for several parameters of interest. Line plots for $T, Y_{\mathrm{CO}_{2}}, Y_{\mathrm{H}_{2} \mathrm{O}}, Y_{\mathrm{CO}}$, and $Y_{\mathrm{OH}}$ are included in Figs. 27-31. For each of these figures, comparisons are made to the baseline RANS data, as well as results of cases IFPV4 and CFPV4, at a spanwise plane through the centerline of the injectors at several streamwise locations through the primary and secondary flames. While the temperature shows agreement with the baseline RANS data similar to that of cases IFPV4 and CFPV4, the results for $Y_{\mathrm{CO}_{2}}, Y_{\mathrm{H}_{2} \mathrm{O}}, Y_{\mathrm{CO}}$, and $Y_{O H}$ show some improvement. Specifically, at most of the streamwise locations, the CFPVX4 results more closely track the baseline RANS data, with the most significant improvement being for $Y_{O H}$. At each of the streamwise locations in Fig. 31, the CFPVX4 results show considerable improvement over both the IFPV4 and CFPV4 cases, and these results are particularly promising due to significant difficulty FPV models typically have predicting minor species concentrations. Inspecting the results for $Y_{\mathrm{CO}_{2}}$ and $Y_{\mathrm{CO}}$ suggests some minor multivaluedness of the CFPVX4 manifold. The spikes in concentration for $Y_{\mathrm{CO}_{2}}$ at measurement locations $24 \mathrm{~cm}$ and $25.5 \mathrm{~cm}$ and for $Y_{C O}$ at location $24 \mathrm{~cm}$ are a result of the slight multivaluedness of the $Z-C-p-h$ manifold mentioned previously. This problem is common to all FPV modeling approaches, since the manifold mappings typically used are generally not completely unique. Further work is necessary to parse the multivalued portions of the flamelet state-space and remove some of the flamelet solutions around the manifold overlap region to help alleviate this problem. 

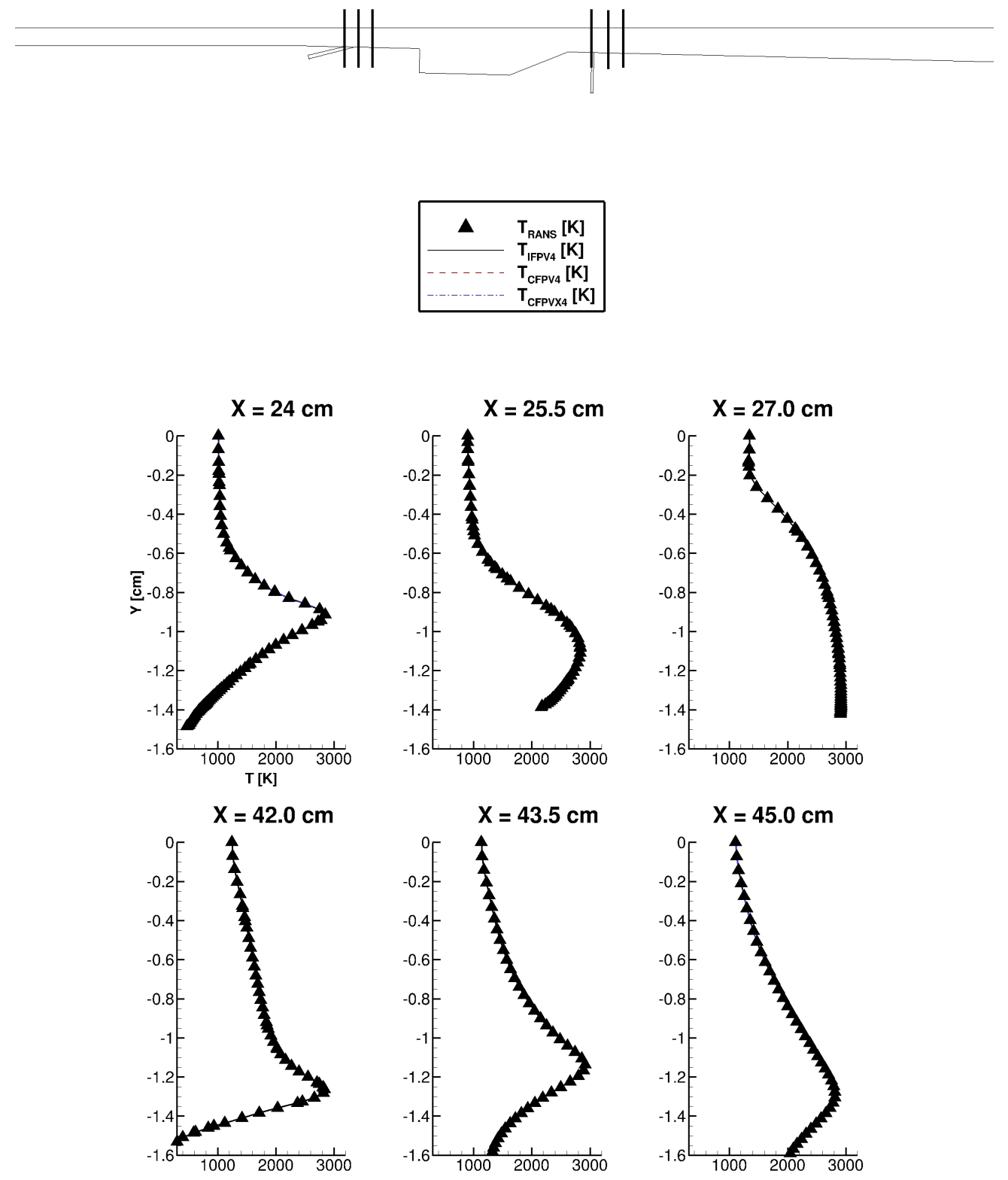

Figure 27. Line plots of $Y[\mathrm{~cm}]$ versus static temperature, $T[\mathrm{~K}]$, obtained using the APFM-RANS analysis method and from the baseline RANS simulations of the HDCR combustor at several locations through the primary and secondary injector flames at the injector centerline nearest to the combustor centerline for cases corresponding to IFPV4, CFPV4, and CFPVX4. 

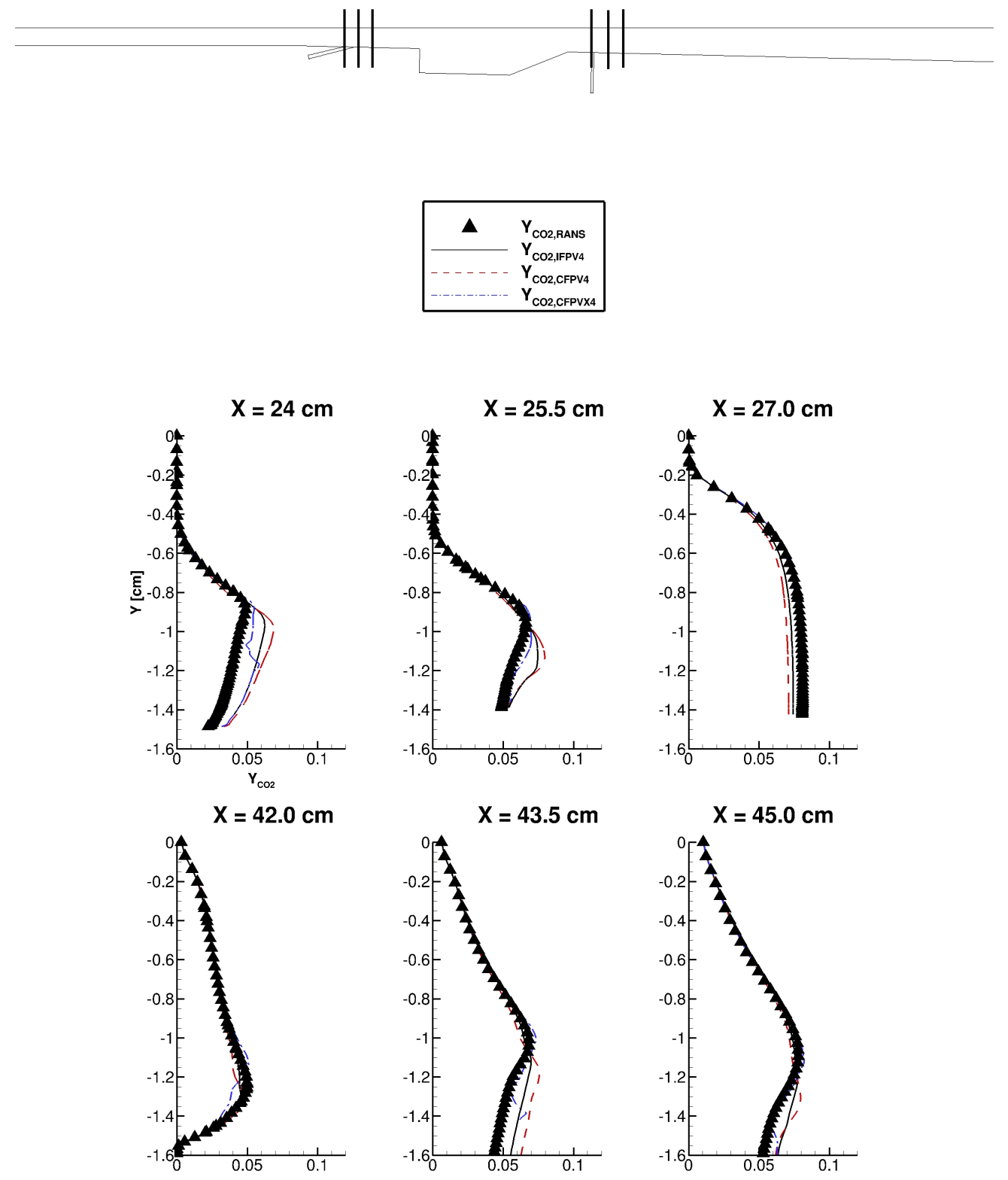

Figure 28. Line plots of $Y$ [cm] versus $Y_{\mathrm{CO}_{2}}$ obtained using the APFM-RANS analysis method and from the baseline RANS simulations of the HDCR combustor at several locations through the primary and secondary injector flames at the injector centerline nearest to the combustor centerline for cases corresponding to IFPV4, CFPV4, and CFPVX4. 

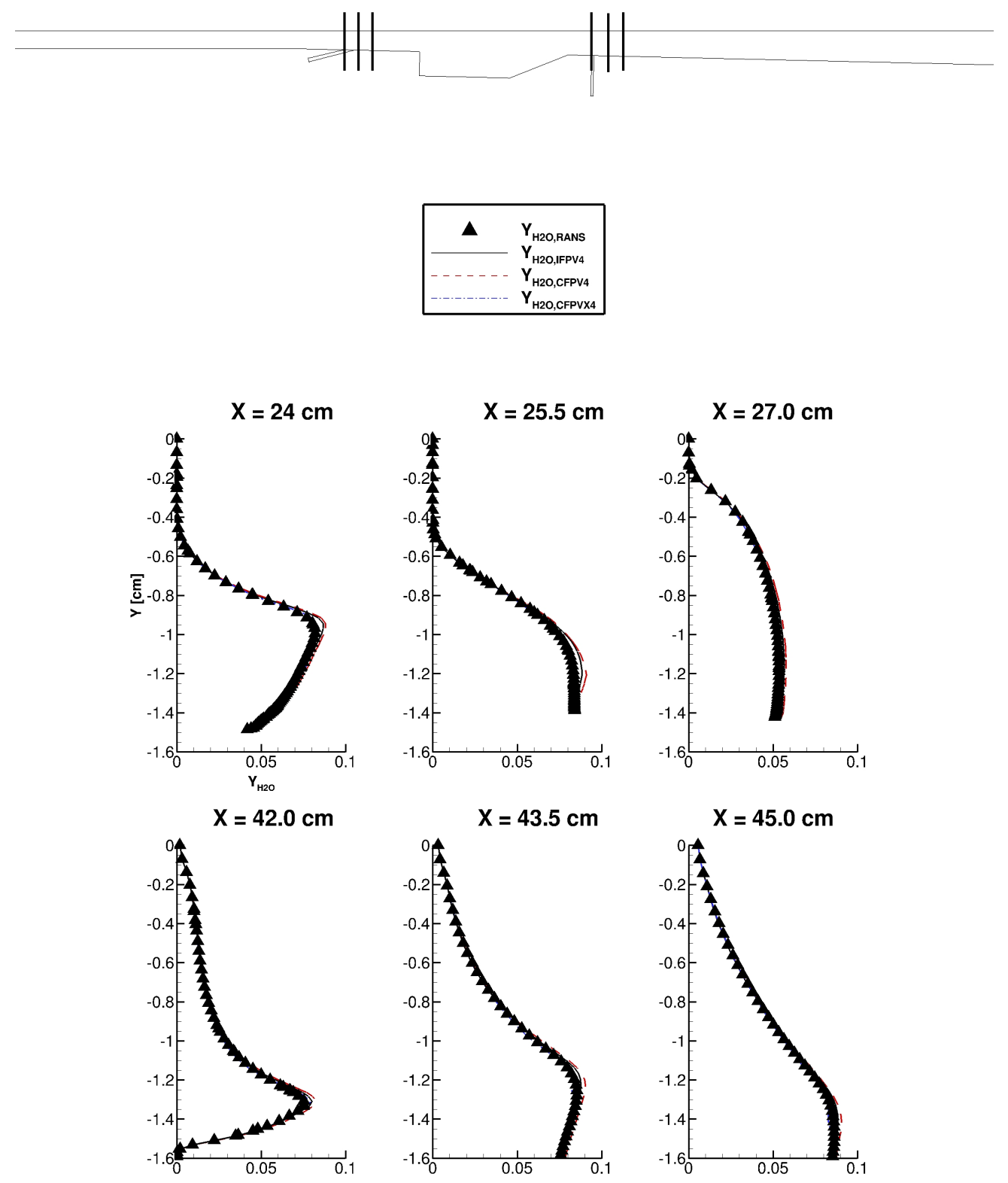

Figure 29. Line plots of $Y$ [cm] versus $Y_{\mathrm{H}_{2} \mathrm{O}}$ obtained using the APFM-RANS analysis method and from the baseline RANS simulations of the HDCR combustor at several locations through the primary and secondary injector flames at the injector centerline nearest to the combustor centerline for cases corresponding to IFPV4, CFPV4, and CFPVX4. 

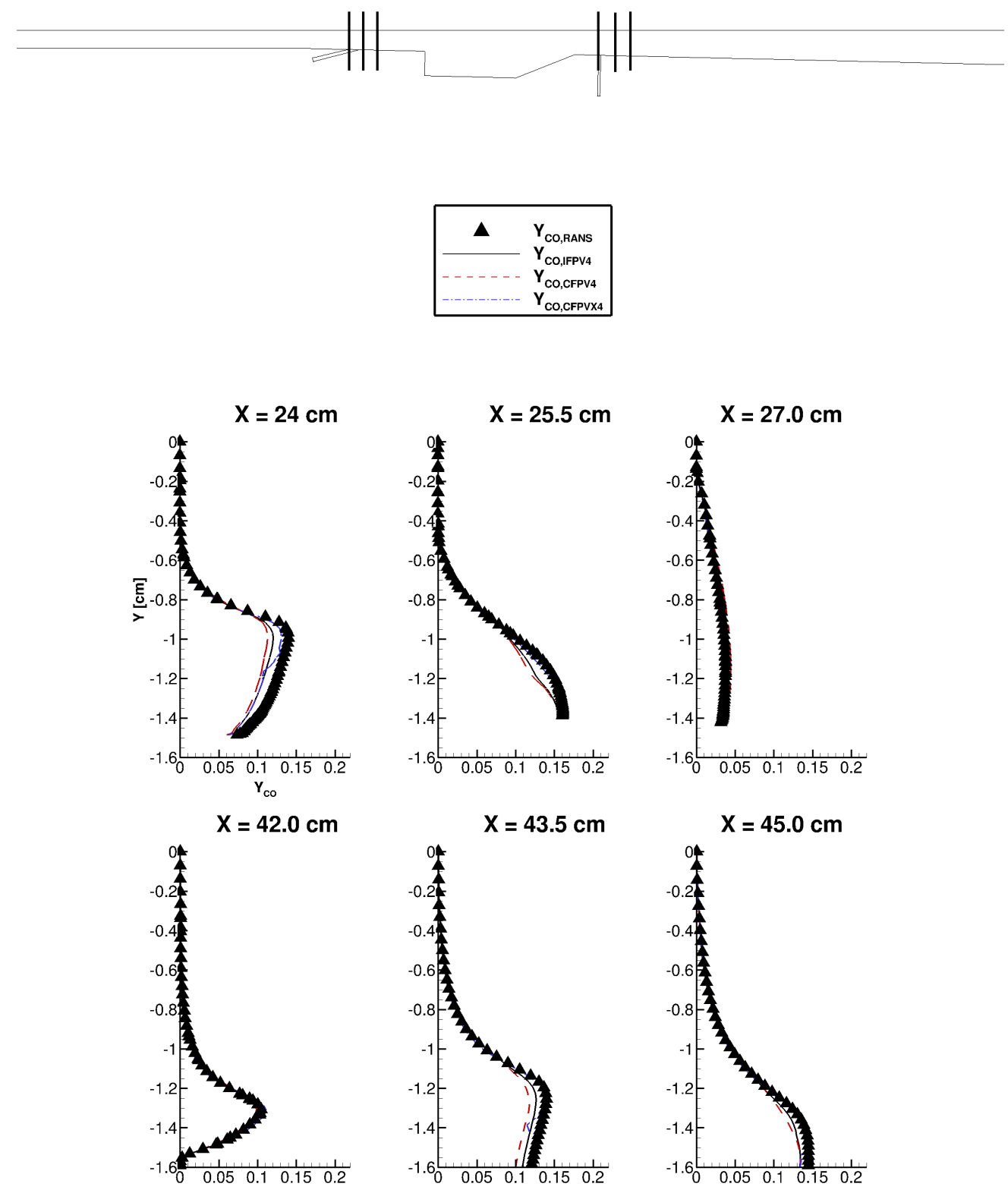

Figure 30. Line plots of $Y$ [cm] versus $Y_{C O}$ obtained using the APFM-RANS analysis method and from the baseline RANS simulations of the HDCR combustor at several locations through the primary and secondary injector flames at the injector centerline nearest to the combustor centerline for cases corresponding to IFPV4, CFPV4, and CFPVX4. 

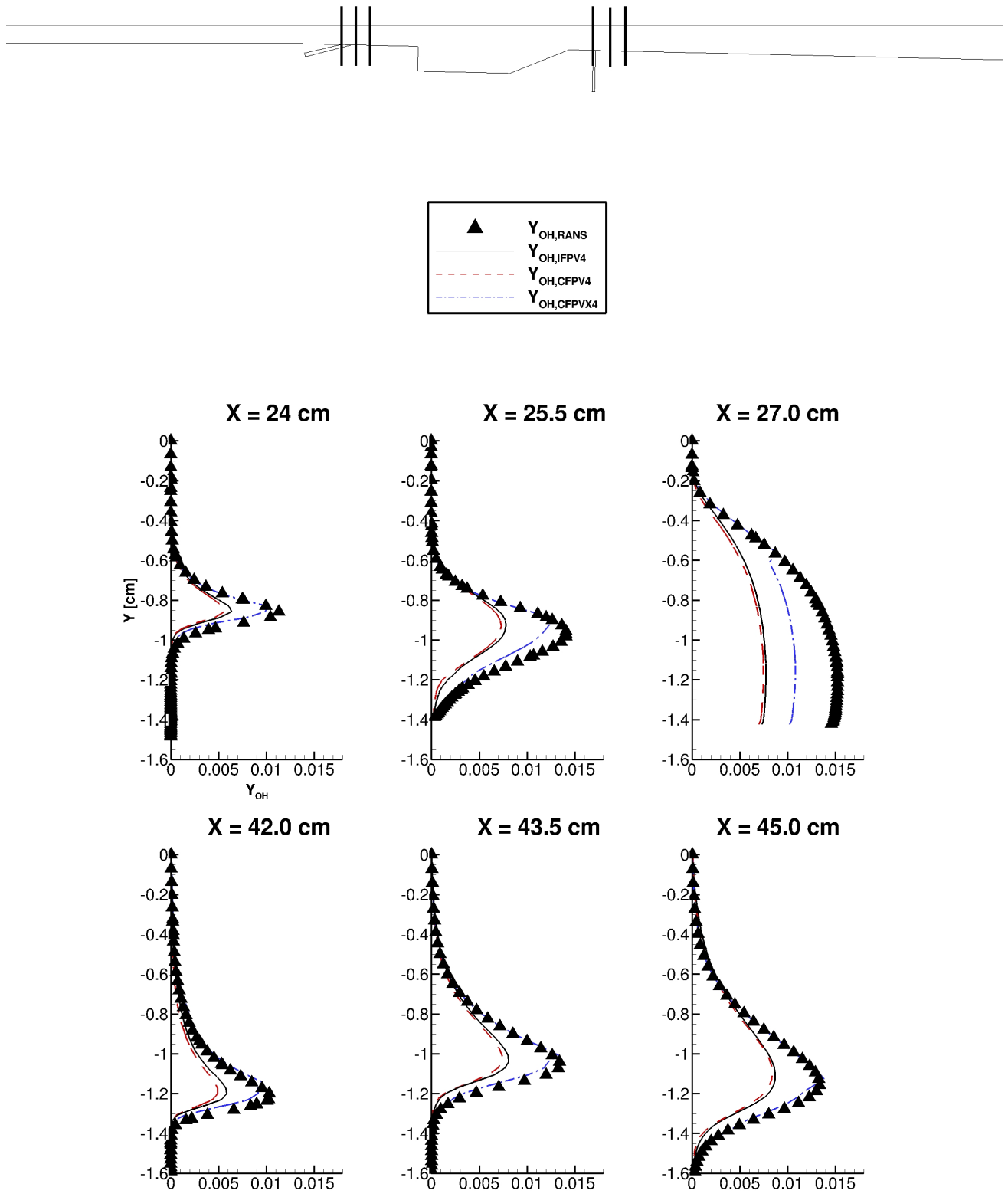

Figure 31. Line plots of $Y$ [cm] versus $Y_{O H}$ obtained using the APFM-RANS analysis method and from the baseline RANS simulations of the HDCR combustor at several locations through the primary and secondary injector flames at the injector centerline nearest to the combustor centerline for cases corresponding to IFPV4, CFPV4, and CFPVX4.

Scatter plots of $T$ versus the baseline RANS data are included in Fig. 32, and similar scatter plot comparisons to the baseline RANS data for $Y_{\mathrm{CO}_{2}}, Y_{\mathrm{H}_{2} \mathrm{O}}, Y_{\mathrm{CO}}$, and $Y_{\mathrm{OH}}$ are included in Fig. 33. These scatter plots show data for the entire HDCR combustor flowfield, similar to the figures shown previously for cases IFPV1-IFPV4 and CFPV1-CFPV4 in Figs. 11-15 and 16-20. However, for Figs. 32-33, the results for case CFPV4 are also plotted in green to highlight the improvements made by the CFPVX4 model. For temperature, little improvement can be seen owing mostly to the good agreement with the baseline RANS data for both the CFPV4 and CFPVX4 cases. Considerably more improvement, however, can be seen in the results for $Y_{\mathrm{CO}_{2}}, Y_{\mathrm{H}_{2} \mathrm{O}}, Y_{\mathrm{CO}}$, and $Y_{\mathrm{OH}}$. In each case, the CFPVX4 results, shown by black points, exhibit less dispersion around the red line than those of the CFPV4 results. For $Y_{O H}$, the improvement appears most significant, as both the approximate slope of the data spread better matches that of the red line and the dispersion is lessened. The results for $Y_{\mathrm{CO}_{2}}$ also show a substantial improvement, while results for $Y_{\mathrm{H}_{2} \mathrm{O}}$ and $Y_{C O}$ show more modest improvements over the CFPV4 results.

Additionally, $\dot{\omega}_{C}$ was calculated for case CFPVX4 and compared to the baseline RANS solution in Fig. 34, 


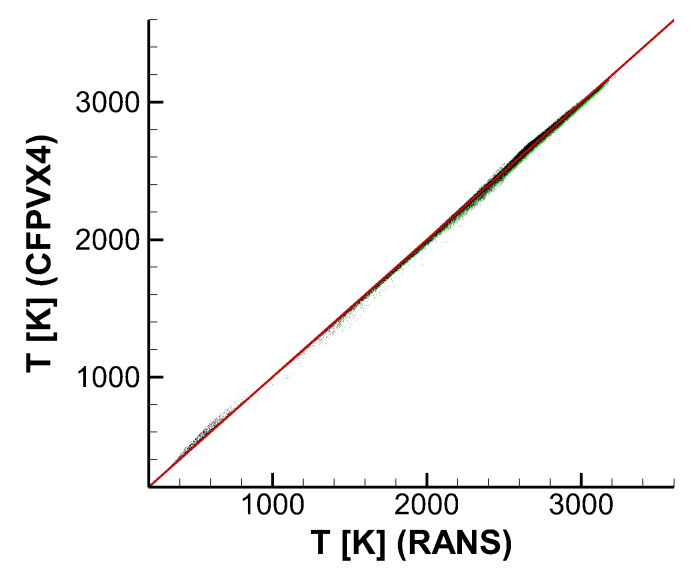

Figure 32. Scatter plots of $T$ obtained using the APFM-RANS analysis method in black versus that from the baseline RANS simulations of the HDCR combustor for the full computational domain for case CFPVX4. Results for case CFPV4 are shown in green, and the red line denotes a line with slope of one.
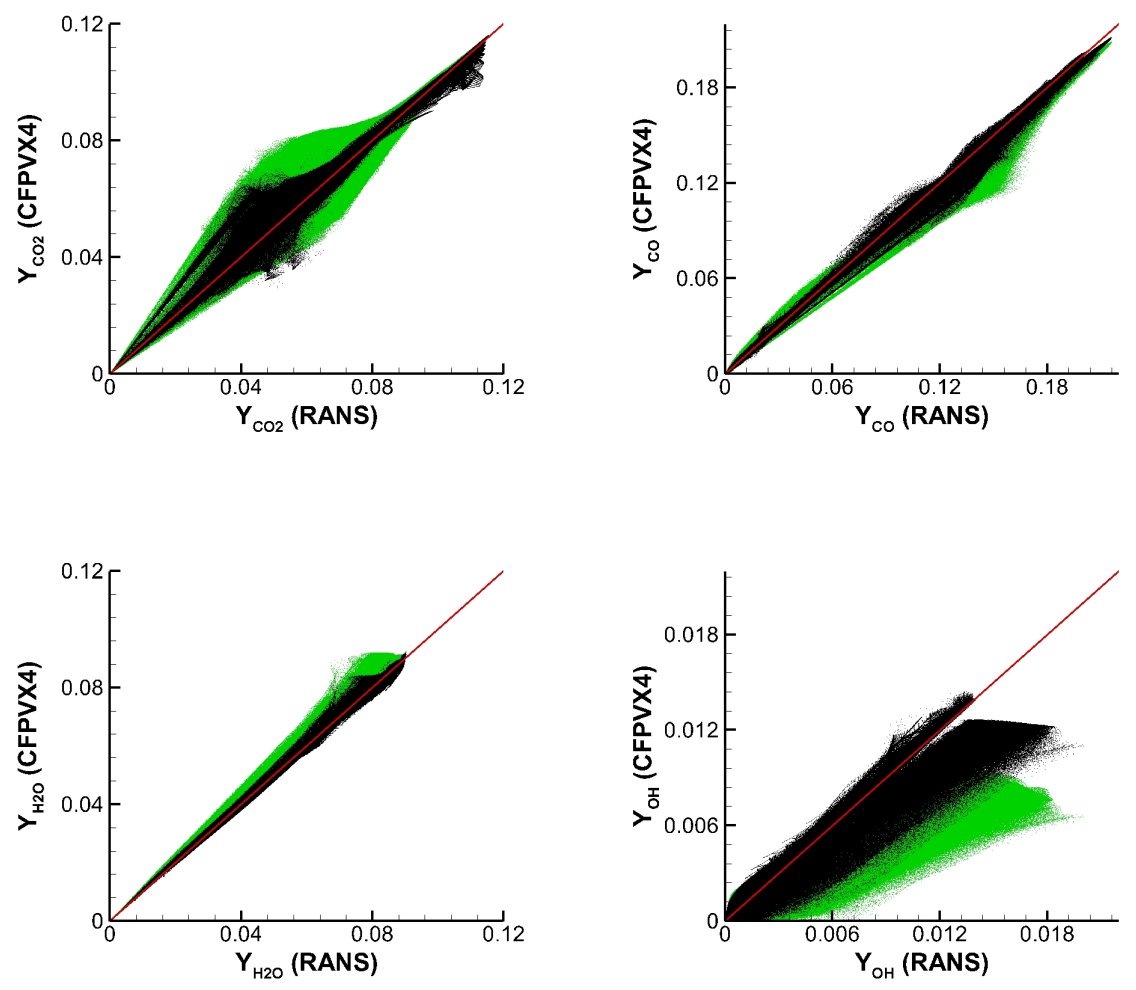

Figure 33. Scatter plots of $Y_{\mathrm{CO}_{2}}, Y_{\mathrm{CO}}, Y_{\mathrm{H}_{2} \mathrm{O}}$, and $Y_{\mathrm{OH}}$ obtained using the APFM-RANS analysis method in black versus that from the baseline RANS simulations of the HDCR combustor for the full computational domain for case CFPVX4. Results for case CFPV4 are shown in green, and the red line denotes a line with slope of one. 
in which the temperature-scaling in Eq. (16) was used. Since case CFPVX4 used FT3, for which pressure was a parameterizing variable, no pressure-scaling was necessary to compute $\dot{\omega}_{C}$. Comparing Fig. 34 to the results for case IFPV4 using Eq. (15) and to case CFPV4 using Eq. (16) in Fig. 23 suggests a similar level of agreement in the prediction of $\dot{\omega}_{C}$ for the CFPVX model. When considered with the results for $T$, $Y_{C O_{2}}$, $Y_{\mathrm{H}_{2} \mathrm{O}}, Y_{\mathrm{CO}}$, and $Y_{\mathrm{OH}}$, the a priori performance of the CFPVX model suggests a modest improvement over the state-of-the-art IFPV and CFPV model formulations for the HDCR combustor.

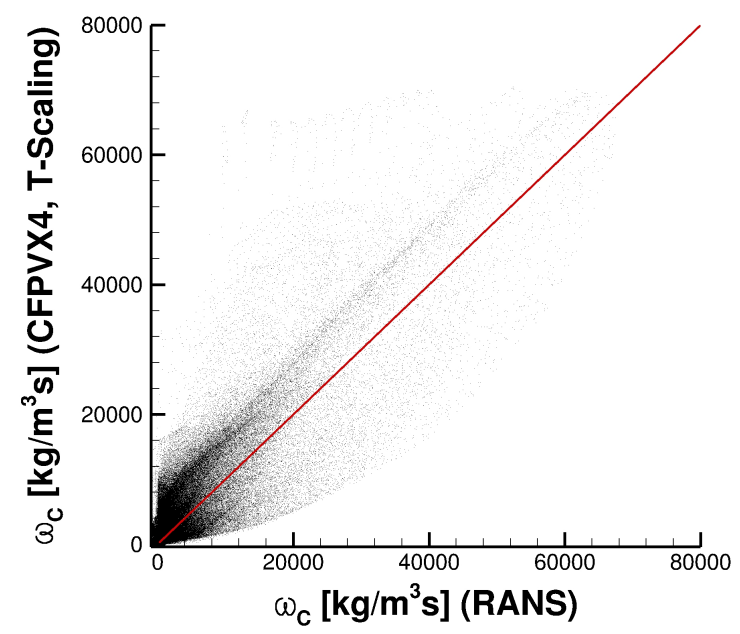

Figure 34. Scatter plot of $\dot{\omega}_{C}$ obtained using the APFM-RANS analysis method for case CFPVX4 versus that from the baseline RANS simulations of the HDCR combustor for the full computational domain. The temperature-scaling presented in Eq. (16) is used to correct the tabulated CFPVX $4 \dot{\omega}_{C}$.

\section{Conclusions and Future Work}

In an effort to assess state-of-the-art FPV model formulations for application to a dual-mode scramjet combustor, a detailed a priori study was performed. RANS simulations of the HDCR dual-mode scramjet combustor were first performed using a 22-species/18-step reaction mechanism and served as the baseline truth solution. The APFM-RANS analysis method was developed for the current study and was used on several FPV model formulations. The data obtained using the APFM-RANS analysis method provided the best-case scenario performance of the FPV models. IFPV and CFPV models were used along with several candidate progress variables, for which a trade study was performed. Based on comparisons of $T, Y_{\mathrm{CO}_{2}}$, $Y_{\mathrm{H}_{2} \mathrm{O}}, Y_{\mathrm{CO}}$, and $Y_{\mathrm{OH}}$, four definitions for $C$ commonly used in the literature were compared, and the most representative $C$ for the current application was found to be $C=Y_{\mathrm{CO}_{2}}+Y_{\mathrm{H}_{2} \mathrm{O}}+Y_{\mathrm{CO}}+Y_{\mathrm{H}_{2}}$.

Using the APFM-RANS analysis method, models used for calculating $\dot{\omega}_{C}$ were also validated. Pressure and temperature scaling techniques, as well as using pressure as an independent parameterizing variable for the flamelet table, were tested. Results suggested that both pressure and temperature effects must be properly accounted for when calculating $\dot{\omega}_{C}$ with an FPV model. The necessity of such an approach is due primarily to the strongly non-linear nature of the $\dot{\omega}_{C}$ term. Further, for compressible flows, the temperature native to the flamelet solutions was found to differ significantly from that of the local transported temperature of the baseline RANS solution. These considerations render the $\dot{\omega}_{C}$ terms from a flamelet table severely incorrect, and appropriate scaling models must therefore be used for a CFPV model formulation.

In addition to $\dot{\omega}_{C}$, the energy interdiffusion term in the total energy transport equation was investigated, as this term serves as an additional coupling to the thermochemical state-space embedded in the flamelet table. Several methods of tabulating and calculating this term were presented and evaluated. Results suggest using a chain rule decomposition of $\frac{\partial Y_{\alpha}}{\partial x_{j}}$ and tabulating only $\frac{\partial Y_{\alpha}}{\partial Z}$ may be a reasonable model for calculating the energy interdiffusion term.

Based on these findings and earlier a priori analysis of the HDCR, a CFPV model was proposed that included the effects of flamelet boundary conditions, which were shown previously to strongly vary across the 
combustor. The CFPVX model extended earlier CFPV model formulations by using $T_{o x}$ to parameterize the flamelet solution space, in addition to using pressure as an explicit parameterizing variable. These developments led to a four-dimensional flamelet table. APFM-RANS analysis results using the CFPVX model were compared to both IFPV and CFPV model results. These comparisons demonstrated an improved agreement with the baseline RANS solution for $T, Y_{\mathrm{CO}_{2}}, Y_{\mathrm{H}_{2} \mathrm{O}}, Y_{\mathrm{CO}}$, and $Y_{\mathrm{OH}}$. When corrected for temperature, $\dot{\omega}_{C}$ also showed good agreement with the baseline RANS solution.

Subsequent work will involve performing APFM-RANS analysis to investigate the applicability of the CFPVX model for scram-mode operation of the HDCR, with subsequent a posteriori validation studies being used to validate related observations and to perform similar CFPVX-modeled RANS simulations. Additionally, future work will involve implementing the CFPVX model in the VULCAN-CFD code and performing a posteriori RANS simulations of the HDCR combustor and comparing the results with those of the current baseline RANS solution.

\section{Acknowledgments}

This work was supported by a NASA Space Technology Research Fellowship (training grant NNX11AM86H) and the NASA Pathways Program, with additional support provided by the Virginia Space Grant Consortium and by a Virginia Commonwealth Fellowship. The authors are grateful for the helpful discussions with Dr. Robert Baurle and Mike Bynum during the course of this study. Computational resources were provided by the NASA Advanced Supercomputing Division.

\section{References}

${ }^{1}$ Peters, N., "Laminar Diffusion Flamelet Models in Non-Premixed Turbulent Combustion," Prog. Energy Combust. Sci., Vol. 10, No. 3, 1984, pp. 319-339.

${ }^{2}$ Peters, N., "Laminar Flamelet Concepts in Turbulent Combustion," Proc. Combust. Inst., Vol. 21, 1986, pp. 1231-1250.

${ }^{3}$ Bekdemir, C., Somers, L., and de Goey, L., "Modeling Diesel Engine Combustion using Pressure Dependent Flamelet Generated Manifolds," Proc. Combust. Inst., Vol. 33, No. 2, 2011, pp. 2887 - 2894.

${ }^{4}$ Terrapon, V. E., Pecnik, R., Ham, F., and Pitsch, H., "Full-System RANS of the HyShot II Scramjet Part 2: Reactive Cases," Annual Research Briefs, Center for Turbulence Research, Stanford University, Stanford, CA, 2010.

${ }^{5}$ Larsson, J., Vicquelin, R., and Bermejo-Moreno, I., "Large Eddy Simulations of the HyShot II Scramjet," Annual Research Briefs, Center for Turbulence Research, Stanford University, Stanford, CA, 2011.

${ }^{6}$ Pope, S. B., "Computationally Efficient Implementation of Combustion Chemistry using in situ Adaptive Tabulation," Combust. Theor. Model., Vol. 1, No. 1, 1997, pp. 41-63.

${ }^{7}$ Esposito, G. and Chelliah, H. K., "Skeletal Reaction Models Based on Principal Component Analysis: Application to Ethylene-Air Ignition, Propagation, and Extinction Phenomena." Combust. Flame, Vol. 158, 2011, pp. 477-489.

${ }^{8}$ Magnussen, B. F. and Hjertager, B. W., "On the Structure of Turbulence and a Generalized Eddy Dissipation Concept for Chemical Reaction in Turbulent Flow," 19th AIAA Aerospace Science Meeting, AIAA, St. Louis, MO, Jan. 1981.

${ }^{9}$ Cabell, K., Hass, N. E., Storch, A., and Gruber, M., "HIFiRE Direct-Connect Rig (HDCR) Phase I Scramjet Test Results from the NASA Langley Arc-Heated Scramjet Test Facility," 17th AIAA International Space Planes and Hypersonic Systems and Technologies Conference, San Francisco, CA, 2011.

${ }^{10}$ Quinlan, J. R., McDaniel, J. C., Drozda, T. G., Lacaze, G., and Oefelein, J., "A Priori Analysis of Flamelet-Based Modeling for a Dual-Mode Scramjet Combustor,", 50th AIAA Joint Propulsion Conference, AIAA, Cleveland, OH, 2014.

${ }^{11}$ Quinlan, J., McDaniel, J. C., Drozda, T., Lacaze, G., and Oefelein, J. C., "A Priori Analysis of Flamelet-Based Modeling for a Dual-Mode Scramjet Combustor," 66th Division of Fluid Dynamics Meeting of the American Physical Society, Pittsburgh, PA, nov 2013.

${ }^{12}$ Pierce, C. and Moin, P., "Progress-Variable Approach for Large-Eddy Simulation of Non-Premixed Turbulent Combustion," J. Fluid Mech., Vol. 504, 2004, pp. 73-97.

${ }^{13}$ Michel, J.-B., Colin, O., and Veynante, D., "Modeling Ignition and Chemical Structure of Partially Premixed Turbulent Flames using Tabulated Chemistry," Combust. Flame, Vol. 152, No. 1-2, 2008, pp. 80 - 99.

${ }^{14}$ Michel, J.-B., Colin, O., Angelberger, C., and Veynante, D., "Using the Tabulated Diffusion Flamelet Model ADF-PCM to Simulate a Lifted Methane-Air Jet Flame," Combust. Flame, Vol. 156, No. 7, 2009, pp. 1318 - 1331.

${ }^{15}$ Knudsen, E. and Pitsch, H., "A general flamelet transformation useful for distinguishing between premixed and nonpremixed modes of combustion," Combust. Flame, Vol. 156, No. 3, 2009, pp. $678-696$.

${ }^{16}$ Pitsch, H. and Ihme, M., "An Unsteady/Flamelet Progress Variable Method for LES of Nonpremixed Turbulent Combustion," AIAA Aerospace Sciences Meeting and Exhibit, Reno, NV, January 2005.

17 "Viscous Upwind ALgorithm for Complex Flow ANalysis (VULCAN)," http://vulcan-cfd.larc.nasa.gov, Nov. 2014.

${ }^{18}$ van Oijen, J. A., Flamelet-generated manifolds: Development and application to premixed laminar flames, Ph.D. thesis, Eidenhoven Technical University, Eidenhoven, Netherlands, 2002.

${ }^{19}$ van Oijen, J. and de Goey, L., "Modeling of Complex Premixed Burner Systems by Using Flamelet-Generated Manifolds," Combust. Theor. Model., Vol. 6, 2002, pp. 463-478. 
${ }^{20}$ Nguyen, P.-D., Vervisch, L., Subramanian, V., and Domingo, P., "Multidimensional flamelet-generated manifolds for partially premixed combustion," Combust. Flame, Vol. In Press, Corrected Proof, 2009, pp. -.

${ }^{21}$ Sadasivuni, S. K., M. W. and Ibrahim, S. S., "Validation of Unsteady Flamelet/Progress Variable Methodology for Nonpremixed Turbulent Partially Premixed Flames," Proceedings of the European Combustion Meeting, 2009.

${ }^{22}$ de Swart, J., Bastiaans, R., van Oijen, J., de Goey, L., and Cant, R., "Inclusion of Preferential Diffusion in Simulations of Premixed Combustion of Hydrogen/Methane Mixtures with Flamelet Generated Manifolds," Flow Turbul. Combust., Vol. 85, 2010, pp. 473-511, 10.1007/s10494-010-9279-y.

${ }^{23}$ Bilger, R. W., Starner, S. H., and Kee, R. J., "On Reduced Mechanisms for Methane-Air Combustion in Nonpremixed Flames," Combust. Flame, Vol. 80, 1990, pp. 135-149.

${ }^{24}$ McBride, B. J., Gordon, S., and Reno, M. A., "Coefficients for Calculating Thermodynamic and Transport Properties of Individual Species," NASA Technical Memorandum 4513, NASA, Cleveland, OH, Feb. 1993.

${ }^{25}$ Pitsch, H., "FlameMaster: A C ++ Computer Program for 0D Combustion and 1D Laminar Flame Calculations," http://www.stanford.edu/ hpitsch/, 1998.

${ }^{26}$ Wilcox, D. C., "Wall Matching, a Rational Alternative to Wall Functions," 27th Aerospace Sciences Meeting, Reno, NV, 1989.

${ }^{27}$ Menter, F. R., "Two-Equation Eddy-Viscosity Turbulence Models for Engineering Applications," AIAA J., Vol. 32, No. 8, 1994, pp. 1598-1605.

${ }^{28}$ Luo, Z., Yoo, C. S., Richardson, E. S., Chen, J. H., Law, C. K., and Lu, T. F., "Chemical Explosive Mode Analysis for a Turbulent Lifted Ethylene Jet Flame in Highly-Heated Coflow," Combust. Flame, Vol. 159, No. 1, 2012, pp. $265-274$.

${ }^{29}$ Storch, A., Bynum, M., Liu, J., and Gruber, M., "Combustor Operability and Performance Verification for HIFiRE Flight 2," 17th AIAA International Space Planes and Hypersonic Systems and Technologies Conference, San Francisco, CA, 2011.

${ }^{30}$ Ihme, M., Shunn, L., and Zhang, J., "Regularization of Reaction Progress Variable for Application to Flamelet-Based Combustion Models," J. Comput. Phys., Vol. 231, 2012, pp. 7715-7721.

${ }^{31}$ Niu, Y., Vervisch, L., and Tao, P., "An Optimization-Based Approach to Detailed Chemistry Tabulation: Automated Progress Variable Definition," Combust. Flame, Vol. 160, No. 1, 2013, pp. 776-785.

${ }^{32}$ Fiorina, B., Baron, R., Gicquel, O., Thevenin, D., Carpentier, S., and Darabiha, N., "Modelling Non-Adiabatic Partially Premixed Flames Using Flame-Prolongation of ILDM," Combust. Theor. Model., Vol. 7, 2003, pp. 449-470.

${ }^{33}$ Fiorina, B., Gicquel, O., Vervisch, L., Carpentier, S., and Darabiha, N., "Approximating the Chemical Structure of Partially Premixed and Diffusion Counterflow Flames Using FPI Flamelet Tabulation," Combust. Flame, Vol. 140, 2005, pp. 147-160.

${ }^{34}$ Fiorina, B., Gicquel, O., Vervisch, L., Carpentier, S., and Darabiha, N., "Premixed Turbulent Combustion Modeling Using Tabulated Detailed Chemistry and PDF," Proc. Combust. Inst., Vol. 30, 2005, pp. 867-874.

${ }^{35}$ Baburic, M., Numerically Efficient Modelling of Turbulent Non-Premixed Flames, Ph.D. thesis, University of Zagreb, 2005.

${ }^{36}$ Perkovic, L., Baburic, M., Priesching, P., and Duic, N., "CFD Simulation of Methane Jet Burner," Proc. of the European Comb. Meeting, 2009.

${ }^{37}$ Ihme, M. and Pitsch, H., "Prediction of Extinction and Reignition in Non-Premixed Turbulent Flames Using a Flamelet/Progress Variable Model. 1. A Priori Study and Presumed PDF Closure," Combust. Flame, Vol. 155, 2008, pp. 70-89.

${ }^{38} \mathrm{Ihme}, \mathrm{M}$. and Pitsch, H., "Prediction of Extinction and Reignition in Non-Premixed Turbulent Flames Using a Flamelet/Progress Variable Model. 2. A Posteriori Study with Application to Sandia Flames D and E," Combust. Flame, Vol. 155, 2008, pp. 90-107.

${ }^{39}$ Saghafian, A., Shunn, L., Philips, D. A., and Ham, F., "Large Eddy Simulations of the HIRiRE Scramjet Using a Compressible Flamelet/Progress Variable Approach," Proc. Combust. Inst., No. 35, 2015.

${ }^{40}$ Hergart, C., Barths, H., and Peters, N., "Using Representative Interactive Flamelets in Three-Dimensional Modelling of the Diesel Combustion Process including Effects of Heat Transfer," Proceedings of the Multidimensional Engine Modeling User's Group Meeting, 2000.

${ }^{41} \mathrm{Wu}, \mathrm{H}$. and Ihme, M., "Modeling of Unsteady Heat Transfer in Flame-Wall Interaction," 66th Division of Fluid Dynamics Meeting of the American Physical Society, Pittsburgh, PA, nov 2013. 